\title{
Early warning for great earthquakes from characterization of crustal deformation patterns with deep learning
}

\author{
J-T. Lin ${ }^{1}$, D. Melgar ${ }^{1}$, A. M. Thomas ${ }^{1}$, and J. Searcy ${ }^{2}$ \\ ${ }^{1}$ Department of Earth Sciences, University of Oregon, Eugene, Oregon, USA \\ ${ }^{2}$ Research Advanced Computing Services, University of Oregon, Eugene, Oregon, USA \\ Corresponding author: Jiun-Ting Lin (jiunting@uoregon.edu)
}

This is a non-peer reviewed preprint submitted to EarthArXiv. This paper has been submitted to the Journal of Geophysical Research - Solid Earth for review. 


\title{
Early warning for great earthquakes from characterization of crustal deformation patterns with deep learning
}

\author{
J-T. Lin'1, D. Melgar ${ }^{1}$, A. M. Thomas ${ }^{1}$, and J. Searcy ${ }^{2}$ \\ ${ }^{1}$ Department of Earth Sciences, University of Oregon, Eugene, Oregon, USA \\ ${ }^{2}$ Research Advanced Computing Services, University of Oregon, Eugene, Oregon, USA \\ Corresponding author: Jiun-Ting Lin (jiunting@uoregon.edu)
}

\section{Key Points:}

- We use synthetic data to train a deep learning model to predict magnitude from crustal deformation patterns in simulated real-time

- The model, M-LARGE, has an accuracy of $99 \%$ and accurately estimates the magnitude of five real large events, outperforming other methods

- M-LARGE's rapid and accurate magnitude prediction suggesting that significant warning times are possible during real large earthquakes

\section{Abstract}

Although infrequent, large (Mw7.5+) earthquakes can be extremely damaging and occur on subduction and intraplate faults worldwide. Earthquake early warning (EEW) systems aim to provide advanced warning before strong shaking and tsunami onsets. These systems estimate earthquake magnitude using the early metrics of waveforms, relying on empirical scaling relationships of abundant past events. However, both the rarity and complexity of great events make it challenging to characterize them, and EEW algorithms often underpredict magnitude and the resulting hazards. Here we propose a model, M-LARGE, that leverages the power of deep learning to characterize crustal deformation patterns of large earthquakes in real time. We demonstrate the algorithm in the Chilean Subduction Zone by training it with more than six million 
27 different simulated rupture scenarios recorded on the Chilean GNSS network. M-LARGE

28 successfully performs reliable magnitude estimation on the testing dataset with an accuracy of $2999 \%$. Furthermore, the model successfully predicts the magnitude of five real Chilean 30 earthquakes that occurred in the last 11 years. These events were damaging, large enough to be 31 recorded by the modern HR-GNSS instrument in the last decade, and provide valuable ground 32 truth. M-LARGE tracks the evolution of the source process and can make faster and more 33 accurate magnitude estimation, significantly outperforming other similar EEW algorithms.

\section{Plain Language Summary}

Great earthquakes are infrequent but devastating natural disasters. To mitigate their effects, earthquake early warning (EEW) systems aim to provide advance warning of strong shaking and tsunami. However, many of the most sophisticated EEW algorithms operating globally have a difficult time characterizing large earthquakes quickly and accurately enough to issue a meaningful warning -- this is most evident from the failure of EEW during the 2011 M9 Tohoku

41 Oki, Japan earthquake. Here we propose a model, M-LARGE, that learns earthquake's surface 42 deformation patterns from millions of simulations, and then apply it to unseen events. Our model 43 shows a high accuracy of $99 \%$ performing on the testing dataset, and accurately estimates the 44 magnitude of five real large historical events in Chile. The M-LARGE outperforms currently 45 operating similar EEW algorithms.

\section{Introduction}

49 Following earthquake initiation, most EEW algorithms provide initial hazard predictions 50 based on the character of the first arriving P-waves, which is the earliest information available. 51 However, it is well known that this approach will routinely struggle during large magnitude 52 earthquakes owing to magnitude saturation, or underestimation, a current limitation of such EEW 
53 systems. As an example of this, the Japanese EEW system mis-identified the $2011 \mathrm{Mw9.0}$

54 Tohoku-oki earthquake as only an Mw8.1 for the first hour after rupture (Hoshiba et al., 2011).

55 Saturation occurs for a couple of reasons. First, inertial-based instruments (seismometers) that

56 record earthquakes in the near-field tend to distort large, low-frequency, signals radiated from

57 large earthquakes, making the data unreliable when real-time automatic processing is considered

58 (Boore \& Bommer, 2005; Larson, 2009; Bock \& Melgar, 2016). Second, methods that directly

59 calculate earthquake magnitude from body waves or surface wave amplitudes focus on particular

60 frequency bands of the source spectrum, which saturate during large (Mw7.5+) events (Geller

61 1976). Third, large earthquakes have durations of several minutes and early onset signals (i.e.

62 the first few seconds of waveforms), utilized by EEW systems, might not contain enough

63 information to forecast the final magnitude of large events (Rydelek \& Horiuchi, 2006; Meier et

64 al., 2016, 2017; Melgar \& Hayes, 2017; Ide, 2019; Goldberg et al., 2019). Magnitude saturation

65 has consequences for downstream applications that rely on rapid magnitude determination,

66 specifically, in the 2011 Tohoku-oki case both forecasts of the expected shaking and the tsunami

67 amplitudes were drastically underpredicted (Colombelli et al., 2013; Hoshiba et al., 2014).

68

69

70

71

72

73

74

In recent years, a number of EEW algorithms have attempted to perform more accurate and faster magnitude calculations. For example, it is possible to match shaking patterns in real-time to the expected geometric extension of the causative fault (Böse et al., 2012; Hutchison et al., 2020). Another approach is to forego complete characterization of the earthquake, and simply take the observed shaking wavefield at a particular instant in time, and forecast its time-evolution into the future (Kodera et al., 2018; Cochran et al., 2019). Furthermore, the advent of widespread high rate global navigation satellite system (HR-GNSS) networks have enabled a new class of EEW algorithms based on measurements of crustal deformation and are particularly well suited to identifying large magnitude earthquakes (Crowell et al., 2013; Grapenthin et al., 2014; Minson et al., 2014; Kawamoto et al., 2016). Noteworthy among these are methods is the Geodetic First 
79 Approximation of Size and Time (GFAST) algorithm which is primarily based on the scaling of 80 peak ground displacement (PGD) and is currently operating in U.S. EEW system for large 81 earthquakes (Crowell et al., 2013, 2016).

More recently, advances in computing power and technologies have enabled the use of machine learning and deep learning algorithms (LeCun et al., 2015). These have also been demonstrated to provide significant improvements in other data-rich seismological applications such as earthquake detection, phase picking, and association (Perol et al., 2018; Ross et al., 2018; Kong et al., 2019; Zhu \& Beroza, 2019; Mousavi et al., 2020b). Earthquake magnitude estimation is a popular application of deep learning, and multiple studies have demonstrated their fast and accurate magnitude prediction (Lomax et al., 2019; Mousavi et al., 2020a; van den Ende \& Ampuero, 2020). of the most advanced EEW systems worldwide (such as the U.S. and Japan) (Murray et al., 2018; Kodera et al., 2020), each of them has limitations. For example, the seismic wavefield-based approaches overcome saturation at the expense of short warning times, typically of the order of 10-20 s (Kodera et al., 2018). Meanwhile, PGD-based approaches avoid saturation but can struggle when earthquakes have very long or unilateral ruptures (Williamson et al., 2020) and can grossly over-predict the magnitudes of these kinds of events. Furthermore, the deep learning algorithms previously proposed that show good performance on magnitude estimation, do not focus on large (Mw7.5+) or even very large (Mw9.0+) magnitude earthquakes. Such events are 101 the most important target of an EEW system. At the root of these difficulties is that every large 102 earthquake is different from the next. Each can, and likely will, have a different starting location, 103 rupture velocity, slip distribution, and radiated seismic energy that evolves in a complex way as 104 the rupture unfolds. All of these properties fundamentally affect EEW system performance and 
105 are difficult if not impossible to predict prior to earthquake occurrence. As such, developing 106 algorithms that can reliably characterize this complexity from surface observations in real-time 107 has proven challenging.

In spite of this diversity of earthquake characteristics, advances in seismic and geodetic 110 instrumentation over the last 30 years have allowed observation and synthesis of the basic 111 kinematic behaviors of large ruptures (Vallée \& Douet, 2016; Ye et al., 2016; Hayes, 2017). 112 Additionally, the location and geometry of the faults on which many large earthquakes are 113 expected to occur are well known (Hayes et al., 2018). By combining these observations, it is 114 now possible to efficiently simulate the rupture process of many potential earthquakes in a realistic 115 way, and to predict their expected seismic and geodetic signatures (Melgar et al., 2016; Frankel 116 et al., 2018; Goldberg \& Melgar, 2020; Pitarka et al., 2020). Another important improvement, 117 specifically in the case of HR-GNSS, is that noise models for real-time data have been proposed 118 (Geng et al., 2018; Melgar et al., 2020). HR-GNSS displacements are a derived product and there 119 can be significant differences between real-time and post-processed solutions. This improvement 120 enables adding noise to any simulated waveform, making the waveforms more realistic.

Here, we will show how to leverage deep learning, the aforementioned earthquake 123 simulations, and their associated HR-GNSS waveforms to characterize earthquake magnitude in 124 real-time. As a demonstration, we apply this approach to the Chilean Subduction Zone which has 125 a dense real-time GNSS network and assess its performance on five recent large-magnitude 126 earthquakes that have occurred there (Figure 1). 

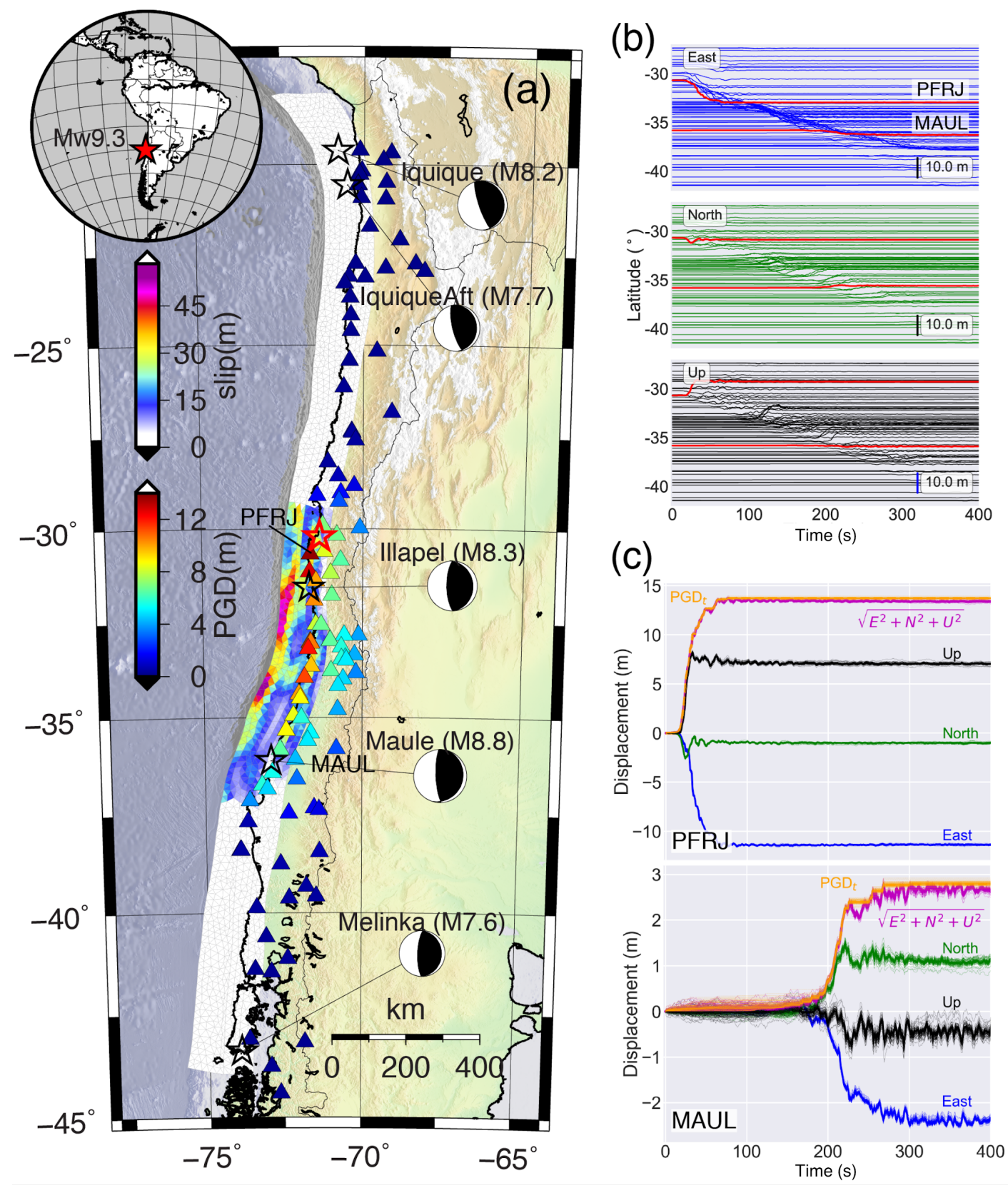

128 Figure 1. Map of the Chilean subduction zone, example rupture scenario, and resulting HR-GNSS waveforms. (a) Slip distribution of a synthetic Mw9.3 earthquake. GNSS stations (triangles) are color coded by their PGD. Focal mechanisms of 5 large events that have occurred since 2010. Red and black stars with focal mechanisms represent the hypocenter of the Mw9.3 rupture scenario and of the historical earthquakes, respectively. (b) Simulated three-component GNSS time series sorted by latitude. Bold red lines denote the records at station PFRJ and MAUL. (c) time series at stations PFRJ and MAUL. Thin lines denote the GNSS noise introduced in the Data and Method section (see section 2.1). 


\section{Data and Methods}

\subsection{Rupture scenarios and synthetic waveforms}

The Chilean Subduction Zone on the west coast of South America is nearly $3000 \mathrm{~km}$ long and accommodates $78-85 \mathrm{~mm} / \mathrm{yr}$ of convergence between the Nazca and South American plates 140 (DeMets et al., 2010). It regularly hosts large magnitude earthquakes including five Mw7.5+ 141 events in the last 10 years (Riquelme et al., 2018). Chile has a real-time HR-GNSS network with more than 120 stations currently in operation (Báez et al., 2018), and provides an excellent testbed for our proposed approach.

For generating the kinematic ruptures we use the Slab2.0 3D slab geometry of (Hayes et al., 2018). We utilize the Chilean slab model from its southern terminus to $\sim 100 \mathrm{~km}$ north of the

Chile/Peru border. We limit the seismogenic depth to $55 \mathrm{~km}$ consistent with the down-dip extent of recently observed large earthquakes (Ruiz \& Madariaga, 2018). The resulting geometry spans a nearly $3000 \mathrm{~km}$ long, $200 \mathrm{~km}$ wide fault. The entire fault is then gridded into a total of 3075 triangular subfaults using a finite element mesher, the average length and width of the subfault vertices is $\sim 12 \mathrm{~km}$.

We generate the 36,800 ruptures on this geometry spanning the magnitude range Mw7.2 to Mw9.4 using the stochastic approach first described by Graves \& Pitarka (2010) with modifications proposed by LeVeque et al., (2016) to avoid the use of Fourier transformations (Figure S1). The magnitudes of the scenarios are uniformly distributed with allowing a small perturbation so that the exact magnitude spans a wider range (Figure S2). The goal here is not 158 to obey the Guttenberg-Richter frequency magnitude distribution but rather to generate a 159 meaningful large and varied number of ruptures to expose M-LARGE to a sufficient variety of 160 sources. The process of generating one particular rupture and its associated waveforms is 
161 described in detail in Melgar et al. (2016) and is summarized here: once the target magnitude is

162 selected, we define the length and width of fault for that particular rupture. We make a random 163 draw from a probabilistic length, L, and width, W, scaling law (Blaser et al., 2010). L and W are 164 obtained from a random draw from the lognormal distributions

\section{5}

166

167

168

169

170

171

172

173

174

175

176

177

178

179

180

$$
\log (L) \sim N\left(-2.37+0.57 M_{w}, \sigma_{L}\right)
$$

with standard deviations defined in the original work of Blaser et al. (2010). The objective is to obtain a length and width that is consistent with the behavior seen in earthquakes worldwide while retaining the observed variability as well. The probabilistic scaling law thus ensures that for a given magnitude we do not always employ the same fault dimensions. Detailed statistics on the resulting fault dimensions for all simulated ruptures can be seen in Figure S1. After the fault dimensions are defined, we randomly select a hypocentral location on the megathrust from a uniform spatial distribution. We do not take into account the variability in along-strike plate convergence rates or any information pertaining to which parts of the megathrust are considered more or less likely to experience a rupture.

Having selected the hypocentral location we next generate the slip pattern and GNSS waveforms. For this we use the Karhunen-Loeve (KL) expansion method (LeVeque et al., 2016, Melgar et al., 2016). The process is separated into the following steps: 1) generate the stochastic slip pattern, 2) define rupture kinematics, and 3) forward model the resulting GNSS waveforms using a Green's function approach. Additional details are provided in Text S1 in the supporting information. Finally, to make the synthetic data more realistic, we introduce noise into the displacement waveform characteristics using a known real-time GNSS noise model (Melgar et 
187 al., 2020) which was computed from analysis of one year-long real HR-GNSS observations

188 spanning a large region. The reference noise model provides expected spectra of noise that vary 189 from the 1st percentile or "low" noise model, continuously through the 50th percentile "median" 190 noise model and up to the 90th percentile "high" noise model. For each waveform we randomly 191 select the percentile noise model and add it to the displacement data. It's worthwhile to note that 192 we only assume the amplitude spectrum of noise, we keep the phase spectrum random. The 193 addition of noise guarantees that the resulting time-domain waveform varies with each realization. 194 In this way we guarantee a large variability of noise and quality in the stations as is routinely seen 195 in true real-time operations.

To ensure that the synthetic waveforms are realistic, we validate the data by comparing the simulated peak ground displacement against what is expected from PGD-Mw scaling (Melgar et al., 2015; Ruhl et al., 2019). This is shown in Figure S3, we find that the synthetic PGD pattern matches the scaling based on real observations at hypocentral distance $\sim 100 \mathrm{~km}$ and $\mathrm{Mw}$ from 201 Mw7.7 to Mw8.7. We note that misfit between modeled and expected values of PGD increases 202 at Mw greater than Mw9.0 or hypocentral distance smaller than $10 \mathrm{~km}$. This is due to the fact that 203 the PGD regressions are constructed from databases of real events; large earthquakes (i.e. 204 Mw9.0+) and very close observations are comparatively rare in those databases (Melgar et al., 205 2016; Ruhl et al., 2019). The large misfit is also due to the limitation of point source assumption 206 in PGD-Mw scaling laws where finiteness of large events need to be considered. All the resulting 207 synthetic rupture scenarios and GNSS waveforms are publicly available on Zenodo 208 (https://doi.org/10.5281/zenodo.5015610) (Lin et al., 2020).

\subsection{M-LARGE : Model architecture}


212 For time-dependent earthquake magnitude prediction we employ a deep learning model,

213 called Machine Learning Assessed Rapid Geodetic Earthquake model (M-LARGE) by linking the

214 input time series recorded at each GNSS station to the time dependent Mw for each rupture

215 derived from integration of the source time function (STF). We attempted to use simple

216 architectures such as artificial neural networks (Figure S4) but ultimately found they did not have

217 enough flexibility to capture the crustal deformation behavior. Our final model is composed of

218 seven fully connected layers and a unidirectional long-short term memory (LSTM) recurrent layer

219 (Hochreiter \& Schmidhuber, 1997), which iteratively predicts Mw using the current and previous

220 HR-GNSS observations across the network (Figure 2; Table S1). We adopted this model

221 architecture because LSTMs are ideal for processing sequential data, which allows M-LARGE to

222 update magnitude predictions as the rupture progresses. Additionally, it does not require a-priori

223 source information (such as the hypocenter) typically required by other rapid modeling methods

224 (e.g. Crowell et al., 2018a). Note that the dense layers only connect the feature values at the

225 same time channel, rather than all the features which would include future times as well. Dropouts

226 are applied to prevent overfitting during the training process (Srivastava et al., 2014). We use a

227 Leaky ReLU function with a slope of 0.1 at negative values (Mass et al., 2013), which is an

228 adaptation of the regular ReLU (i.e. slope of 0 at negative values) (Glorot et al., 2011) for the

229 activation of dense layers. Finally, the last layer is connected to a ReLU function to output a

230 current magnitude prediction, and the goal is to minimize the mean square error (MSE)

231 contributed from the magnitude misfits at every epoch (Figure 2). 

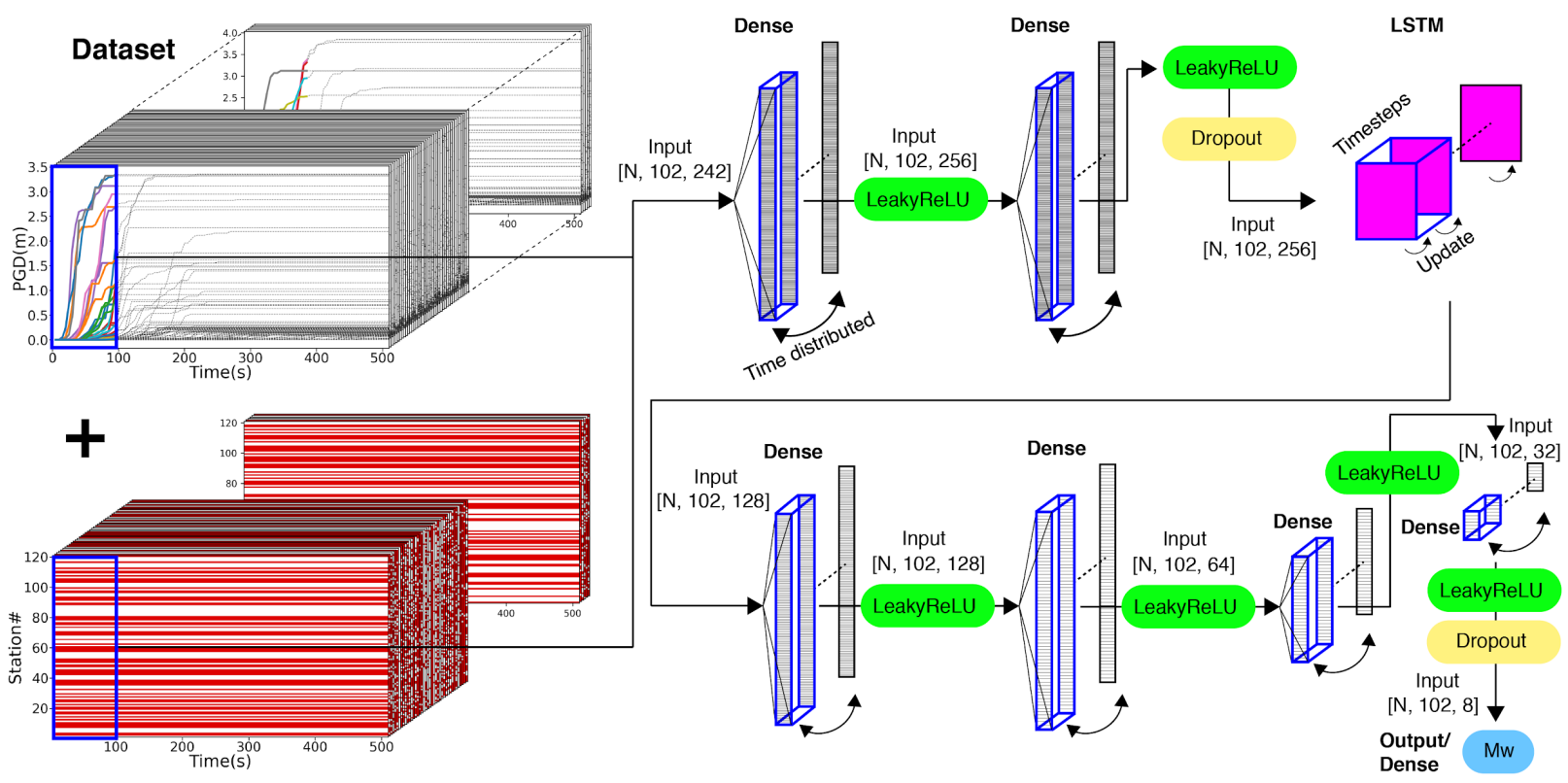

Figure 2. M-LARGE model architecture showing the input as the time-dependent PGD values from the

GNSS stations plus the station on or off (existence) codes. Detailed parameter values are listed in Table

S1. Blue rectangles mark the input PGD time series (i.e. 100 s) from all the available stations with their existence codes, and the participating layers.

\subsection{M-LARGE : Input features, output labeling and model training}

To train the model, we use the 36,800 synthetic ruptures described in section 2.1 and split them into training ( $70 \%$ or 25,760 ruptures), validation ( $20 \%$ or 7,360 ruptures) and testing data

241 (10\% or 3,680 ruptures) (Figure S2). The separation of validation and testing data ensures that the final model, which is selected based on the minimum validation loss, will not be biased during the model testing stage. Note that these are the number of rupture scenarios, the actual input

244 data consider different station recording combination and GNSS noise. In our case, we generate

245 more than 6 million training data from the original training dataset, and 8,192 testing data from 246 the testing dataset (i.e. each rupture has $\sim 2$ different station recording combination and GNSS 247 noise). 


$$
P G D(t)=\max \left(\sqrt{E(t)^{2}+N(t)^{2}+Z(t)^{2}}\right),
$$

253 where $E(t), N(t), Z(t)$ represents the East, North and vertical component of the GNSS 254 displacement time series starting from the earthquake origin (i.e. $t=0$ ), respectively. We use PGD 255 because it has a clear relationship to earthquake magnitude (Crowell et al., 2016; Melgar et al., 256 2015; Ruhl et al., 2019). We introduce feature scaling, which is commonly applied in machine 257 learning, to avoid large feature values dominating smaller ones, making the model convergence 258 difficult. The PGD time series is first clipped at a minimum of $0.01 \mathrm{~m}$ and scaled logarithmically.

259 This is done so that during this re-scaling process the zero-valued data do not diverge to negative 260 infinity. For the model output, we use the time integration from the real STF, convert it to the 261 moment magnitude scale, and re-scale this by multiplying the value by 0.1 for computational 262 efficiency. Both the input and output time series are decimated to 5 second sampling so that we 263 obtain Mw updates in 5 second increments.

To increase the variability of the data, we apply data augmentation by introducing realistic HR-GNSS noise as described in section 2.1. We also randomly select some GNSS records to discard to simulate station outages and network variability yielding more than 6 million earthquake and station scenarios used for 50,000 training steps (Figure S5). The station incompleteness is necessary to simulate station outages which commonly occurs in real-time. For a given network not all stations are operational all of the time, the algorithm should be resilient to this. To

271 distinguish between existing and non-operational stations, we add an additional "station 272 existence" feature channel for every site. We set the value to zero to simulate a station outage, 273 and set it to 0.5 if the station is working normally. This last value is arbitrary, but it is a good 
274 practice to set it to the same scale of other features (i.e. In our feature, 0.5 is $3.16 \mathrm{~m}$ in the original 275 scale, so that their values are comparable).

A total of 121 stations (Figure 1) and 102 time steps (i.e. 5 s sampling for $510 \mathrm{~s}$ of signal 278 duration) of data are employed. Data incompleteness is included by randomly removing stations 279 up to a maximum of 115 stations (i.e. a minimum of only 6 stations remaining). We also set a 280 minimum training threshold of Mw7.5 and require at least 4 stations locate within 3 degrees of the 281 hypocenter. This is to make sure that the training data still carries some clear near-field information, otherwise the algorithm could introduce a bias because of the similar far-field values but different labeled magnitudes. Note that the hypocenter is the only necessary information for the data augmentation step. During the training or actual running process, no hypocenter information is needed. Here we also note that M-LARGE does not detect the onset of an event because our intention here is not to build a detection algorithm, but to determine the correct magnitude when an earthquake is obviously detected. GNSS data is usually noisy enough that event detection from the real-time data can lead to many false positives (Kawamoto et al., 2016). Rather M-LARGE requires triggering, ostensibly by a seismic system as is common in other GNSS algorithms (e.g. Crowell et al., 2018a). The noise in GNSS data is greater than that in seismic data and many algorithms have been demonstrated for detection of the onset of events using inertial recordings (Perol et al., 2018; Ross et al., 2018; Zhu \& Beroza, 2019) so a system that relies on seismometers for triggering is still the most robust.

One assumption we make is that there is no travel time delay due to the propagation of seismic waves from source-to-station in the feature and label pairs. Given the proximity of the

297 Chilean subduction zone to the Chilean GNSS network (Figure 1), we assume any rupture would 298 be recorded soon after the origin. In fact, in most events of our simulations the first arrival occurs 299 by $20 \mathrm{~s}$, and only $6 \%$ of rupture scenarios have arrivals later than $20 \mathrm{~s}$. The latency is only a small 
300 fraction of the $510 \mathrm{~s}$ time series duration. Finally, we save the training weights every 5 epochs

301 and use the model which has the minimum validation loss as the final model (Figure S5). The

302 code base is publicly available and can be obtained at https://doi.org/10.5281/zenodo.4527253

303 (Lin, 2021).

\subsection{Comparison to other geodetic EEW algorithms}

Our main point of comparison for assessing whether M-LARGE is an improvement will be GFAST (Crowell et al., 2016), which predicts magnitude from GNSS observations, and it is also one of the most stable GNSS EEW methods currently operating in the U.S. EEW system (i.e. ShakeAlert). It uses the PGD observations from HR-GNSS time series. When a hypocenter is

311 confirmed by a seismic method, the magnitude is calculated based on the PGD-Mw scaling relationship (Crowell et al., 2016; Melgar et al., 2015; Ruhl et al., 2019). To ensure the data contain PGD information and not noise, a $3 \mathrm{~km} / \mathrm{s}$ travel-time filter is added into the algorithm, and the model only predicts $\mathrm{Mw}$ when at least 4 stations have valid information.

GFAST is not the only GNSS modeling approach; there are other proposed algorithms that 317 utilize near-field GNSS data to rapidly estimate earthquake magnitude. To further compare with 318 M-LARGE we also run the Global Positioning System based centroid moment tensor (GPSCMT) 319 method, which utilizes the near-field static offset term from the GNSS records to calculate 320 magnitude, moment tensor and centroid location (Melgar et al., 2012; Lin et al., 2019). Unlike the 321 GFAST approach, GPSCMT does not require hypocenter information, instead it grid-searches 322 every pre-defined centroid location and solves for the moment tensor. We take the same subfault 323 meshes used by M-LARGE as the potential centroid locations for the GPSCMT algorithm. Both 324 the performance of GFAST and GPSCMT are shown in the next section. 
$327 \quad 3$ Results

$328 \quad 3.1$ M-LARGE performance on testing dataset

329 The performance of M-LARGE on the testing dataset is shown in Figure 3. To quantify how 330 well the model performs on testing data, we define a correct prediction as one within $+/-0.3$ units 331 of the target magnitude (i.e. time-dependent magnitude) and calculate the model accuracy (Figure $3323 b)$. Within these bounds, the model performs well with a high accuracy of $95 \%$ after $60 \mathrm{~s}$ which 333 increases to $99 \%$ by $120 \mathrm{~s}$. The standard deviation of the magnitude misfits are $0.15,0.1,0.09$ at 60,120 , and $360 \mathrm{~s}$, respectively. The accuracy change from $120 \mathrm{~s}$ to $360 \mathrm{~s}$ is not significant; however, this additional time improves some underestimations of long source duration events with magnitude greater than Mw8.5 (Figure 3a).

We compare this statistic to the GFAST algorithm by using the same testing dataset as MLARGE. Note that for GFAST, we remove those predictions with $\mathrm{Mw}=0$ due to the four-station minimum and only show the data that have non-zero values (Figure $3 b$ ). In this example, over $30 \%$ of the testing events have $\mathrm{Mw}=0$ prediction at $60 \mathrm{~s}$, these predictions require additional time to converge compared to our model. Despite this removal, we find that GFAST has a lower accuracy of $62 \%$ at $60 \mathrm{~s}$ which slowly increases to $78 \%$ by $120 \mathrm{~s}$, and to $80 \%$ by $360 \mathrm{~s}$. In

344 comparison to M-LARGE's maximum accuracy of $99 \%$, GFAST's accuracy saturates at $86 \%$ by 345215 seconds. The standard deviation of the magnitude predictions of GFAST are also larger, 346 which are $0.22,0.21,0.22$ at $60,120,360 \mathrm{~s}$, respectively, about 2 times more scatter than the M347 LARGE performance. To summarize, M-LARGE reaches $80 \%$ accuracy 5 times faster than 348 GFAST and has half the scatter on average. 
$41 \%, 28 \%$ and $28 \%$ at $60,120,360$ s, respectively. Noting that the GPSCMT performs with overall

353 much larger scatter, lower accuracy, and systematic overestimations. This has been noted before,

354 that a point source has limited ability on recovering the deformation of large offshore events (e.g.

355 Melgar et al., 2013). Thus, without additional constraints, the model accuracy of GPSCMT method 356 is about $40 \%$ according to our testing dataset.

(a)
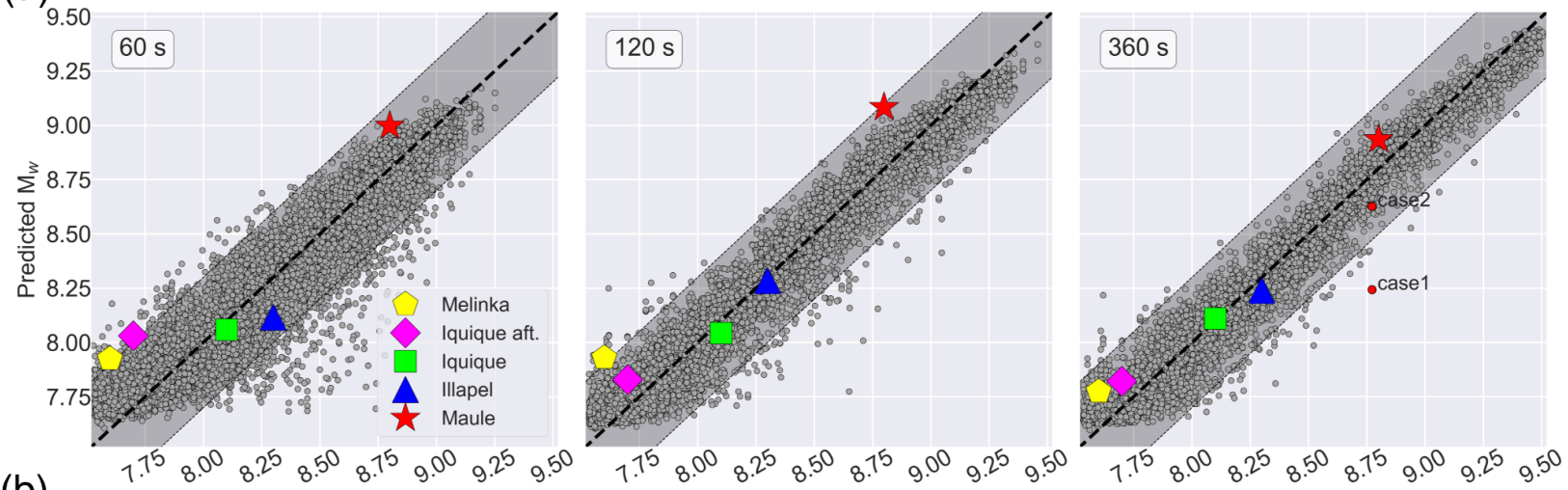

(b)
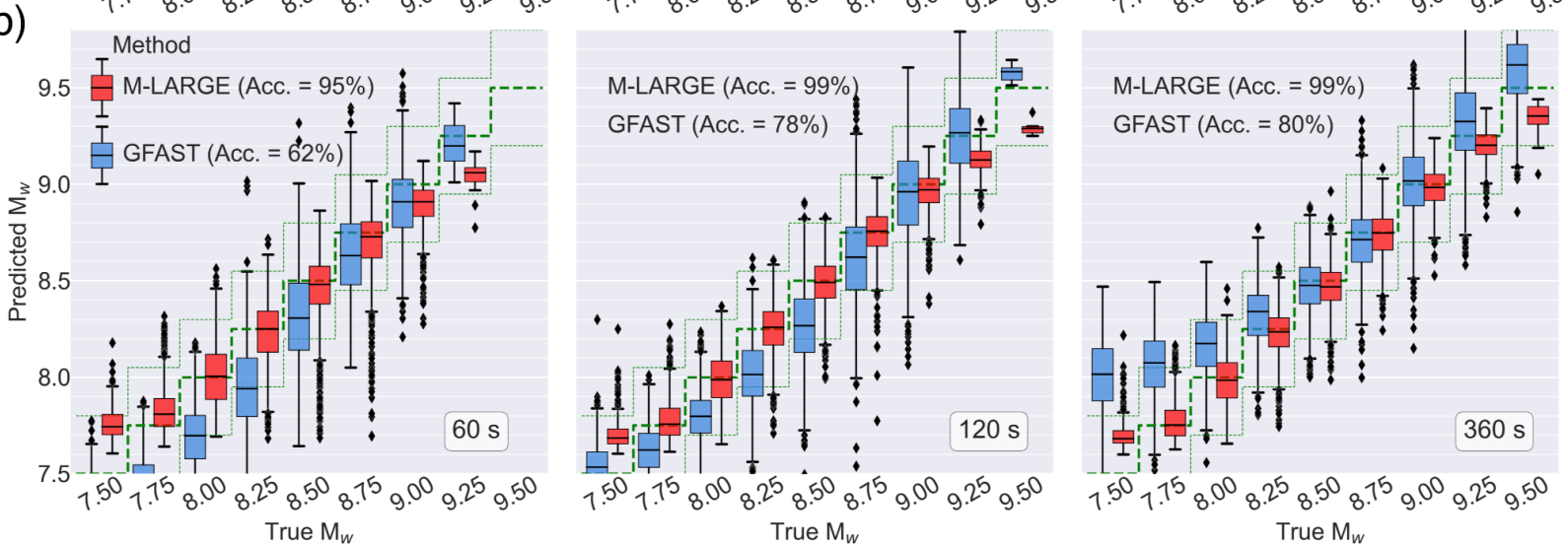

Figure 3. Model performance on testing dataset and on real events. (a) (from left to right) snapshots of MLARGEs performance at 60, 120, 360 s, respectively. Gray dots show the Mw predictions compared to the time-dependent magnitude. Black dashed line represents the 1:1 line; shaded area represents the \pm 0.3 magnitude range. Colored markers denote the M-LARGE predicted $M w$ and their final $M w$ for 5 real 
3.3. (b) comparison of the GFAST (blue) and M-LARGE (red) predicted magnitudes at 60,120 and $360 \mathrm{~s}$ for different magnitude bins. Model accuracies at 60,120 and $360 \mathrm{~s}$ are shown in text. The thick and thin green dashed lines show the 1:1 and \pm 0.3 reference for each magnitude bin.

\subsection{M-LARGE performance on real large earthquakes}

To further assess the performance of M-LARGE, we apply the model to five large historical events in the Chilean Subduction Zone with HR-GNSS records which were not used for training (Figure 4). For each of these earthquakes, different numbers of GNSS sites were available. For the 2010 Mw8.8 Maule earthquake the model only takes $40 \mathrm{~s}$ to reach the $+/-0.3$ magnitude unit criteria. M-LARGE also successfully predicts the final magnitude of the 2014 Mw8.1 Iquique and the 2015 Mw8.3 Illapel earthquakes at $20 \mathrm{~s}$ and 60 s, respectively. For the 2014 Mw7.7 Iquique aftershock and the 2016 Mw7.6 Melinka earthquakes, both the M-LARGE predictions slightly overshoot the true magnitude at $30 \mathrm{~s}$, but soon correct downward to their actual magnitude ranges. Compared to the performance of GFAST, which underestimates the Mw7.7 lquique aftershock by 0.3 magnitude units and overestimates the Melinka earthquake by 0.6 magnitude units, our model shows more robust results on those smaller magnitude events where large GNSS noise dominated the data across the whole network spanning a $3000 \mathrm{~km}$ long subduction zone (Figure 1). We also note that the performance is bounded by the delay times prior to the P-wave arrival at the closest stations. For example the Maule earthquake, where most of the presently operating closest stations did not exist in 2010, the first arrival time was $17 \mathrm{~s}$. Considering these delay times, useful predictions are made as soon as the signals are recorded but the lowest uncertainties are anticipated after $\sim 30 \mathrm{~s}$. The timing of the successful prediction correlates with the source duration, data sparsity or coverage, and the signal-to-noise ratio of the event, which will be further discussed in section 3.3, 3.4, and in the discussion section. 

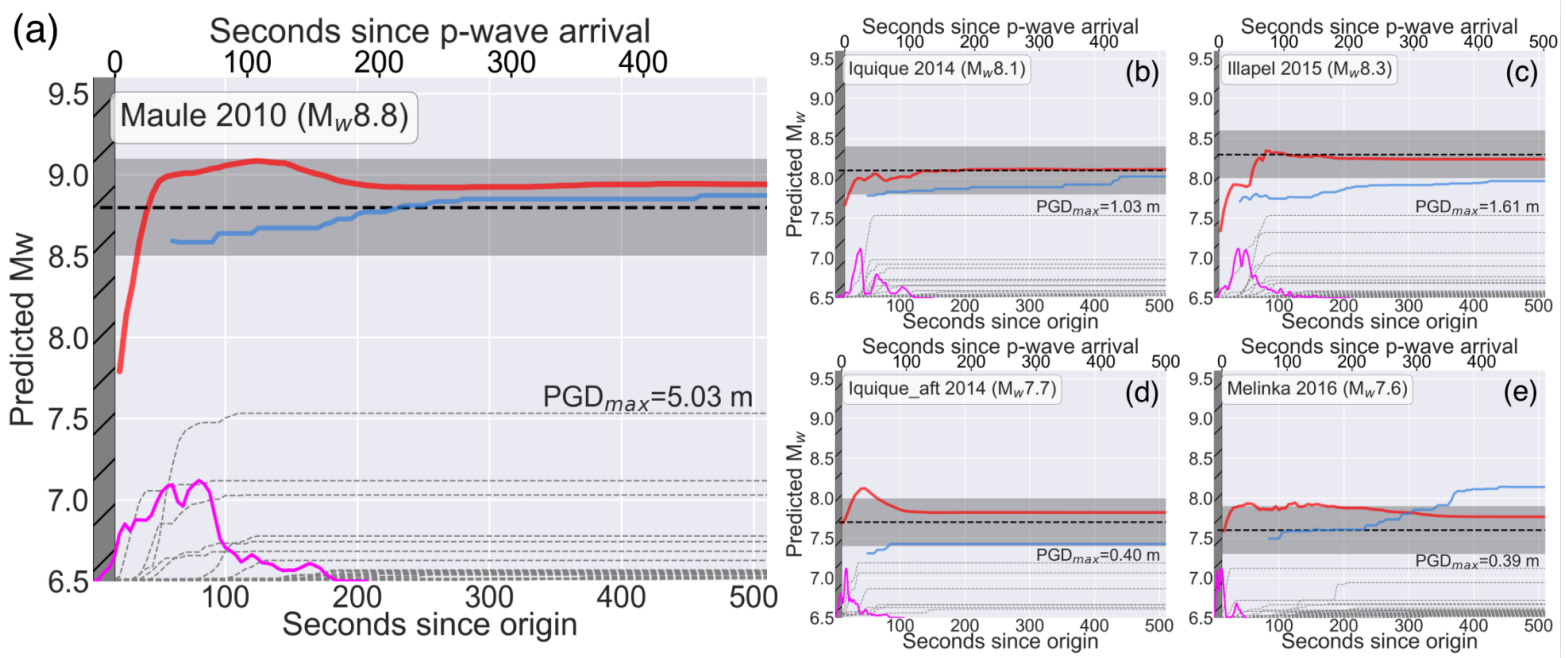

Figure 4. M-LARGE performance on real Chilean earthquakes compared to GFAST. (a) The 2010 Maule

Mw8.8 earthquake. Red and blue line shows the M-LARGE and GFAST prediction, respectively. Black

dashed line and gray shaded areas represent the true $M w$ and the +/-0.3 magnitude unit range. Thin dashed

lines show the PGD waveforms from the 2010 Maule earthquake (Figure 1). Magenta line represents the event source time function from the USGS finite fault product. Hatched dark gray area is the time period prior to the arrival of the P-wave at the closest site where no information on the rupture is available. (b)-(e)

Same as (a) but for the 2014 Mw8.1 Iquique earthquake, the 2015 Mw8.3 Illapel earthquake, the 2014 Mw7.7 Iquique aftershock, and the 2016 Mw7.6 Melinka earthquake, respectively.

\subsection{M-LARGE testing: Imperfect or sparse data}

To understand how M-LARGE performs on imperfect data, we test M-LARGE on two different recording scenarios of the same rupture from the testing dataset. One scenario has poor station coverage whereas the other has excellent station coverage (i.e. red dots in Figure 3a) (Figure 5). In the poor station coverage example (i.e. Case 1 in Figure 5), almost all the near-field data are missing and M-LARGE fails to estimate the true magnitude. It is not until far-field stations begin recording data that M-LARGE upgrades its moment estimate closer to the lower bound of the 
411 near-field data is used to accurately characterize the rupture process and M-LARGE predicts the 412 actual magnitude successfully (Figure 5b, 5d). This suggests that data sparsity in the near-field 413 plays an important role for the accuracy and timeliness of the predictions. The clear implication is 414 that having more stations closer to the source improves M-LARGE's performance.

415

416

417

418

419 

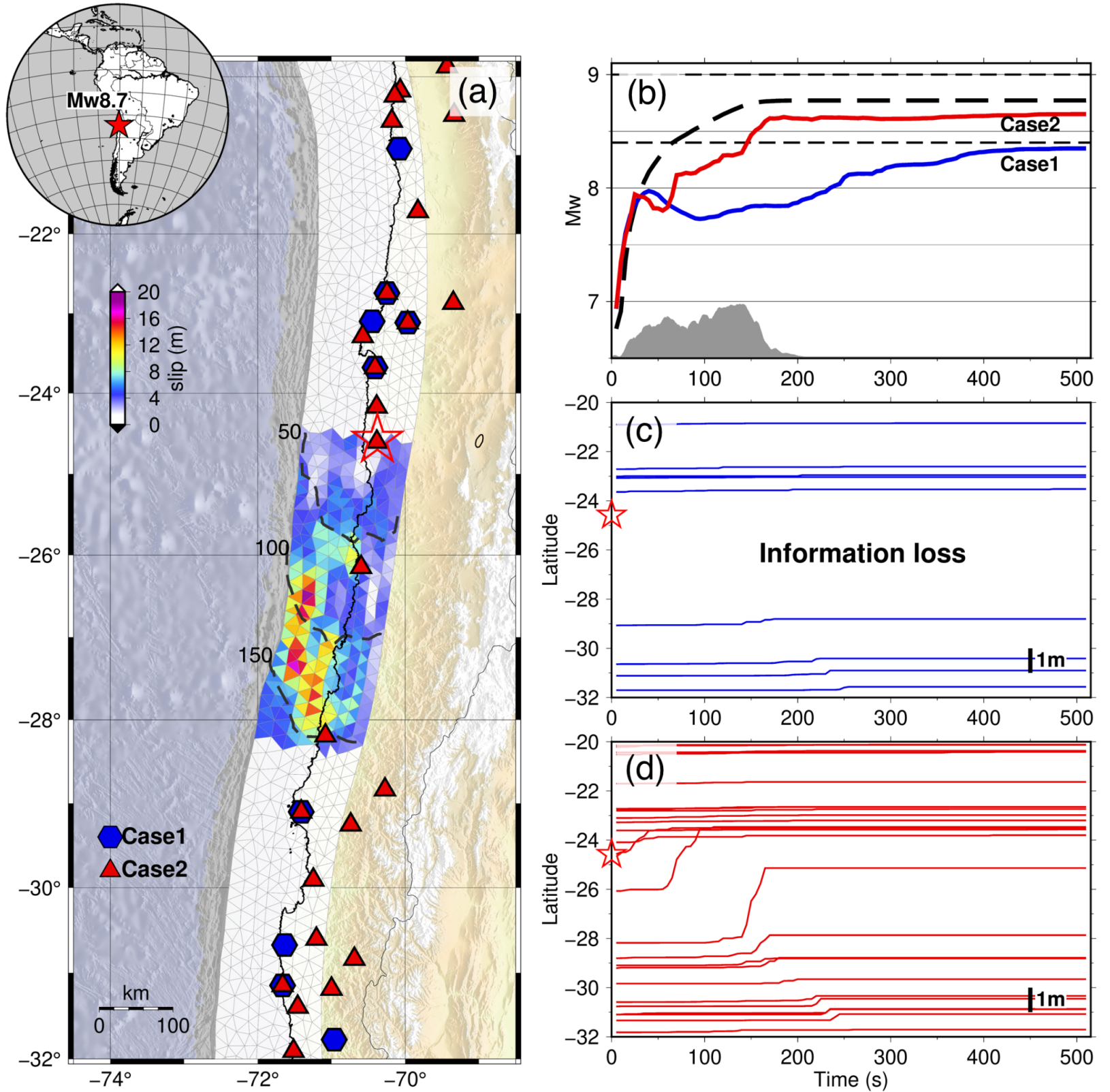

Figure 5. M-LARGE prediction tests with two different station distributions. (a) rupture scenario of a Mw8.7

422 earthquake with the station distribution of Case1 (blue hexagon) and Case2 (red triangle). Red star denotes

423 the hypocenter. Black dashed lines show the 50 s rupture time contours. (b) M-LARGE predictions for Case

4241 (blue line), Case 2 (red line) and the actual Mw (dashed line) calculated from the STF (gray area). (c)

425 PGD data of Case 1 sorted by latitude, Red star denotes the hypocenter latitude. (d) similar to (c), but data 426 of Case 2. 


\section{$429 \quad 3.4$ M-LARGE testing: STF complexity}

430 To understand M-LARGEs performance as a function of source complexity, we choose four 431 different characteristic source time function shapes (i.e. symmetric, bimodal, early and late 432 skewed) and analyze the results. Figure 6 shows examples of each of these characteristic STFs. 433 The models successfully converge to their actual magnitude. We use the time-to-peak STF 434 (hereafter, peak time $\tau_{p}$ ) and centroid time $\left(\tau_{c e n t}\right)$ as proxies to measure the model performance 435 on different STF shapes. We define the time to corrected prediction $\left(\tau_{c}\right)$ as the time when the 436 model successfully predicts the final magnitude with a misfit smaller than 0.3 (Figure 6). We 437 calculate the correlation coefficient (CC) between these metrics (Figure S7) and find that the $\tau_{c}-$

$438 \tau_{p}$ has the weakest correlation with $\mathrm{CC}=0.66$ compared to $\tau_{c}-\tau_{\text {cent }}$ of $\mathrm{CC}=0.9$ and $\tau_{c}-\tau_{\text {duration }}$ 439 of $\mathrm{CC}=0.85$. This suggests that the $\tau_{p}$, a proxy of STF's shape, does not significantly affect the 440 timing and accuracy of the M-LARGE estimations. On the other hand, the performance relies on 441 the actual moment release because it is trained to map the STF directly. We note that the CC of $442 \tau_{c}-\tau_{\text {cent }}$ is slightly higher than the $\tau_{c}-\tau_{\text {duration }}$, this is because of the effect of the tolerated 443 magnitude which we will discuss in section 4.2 . 
(a)

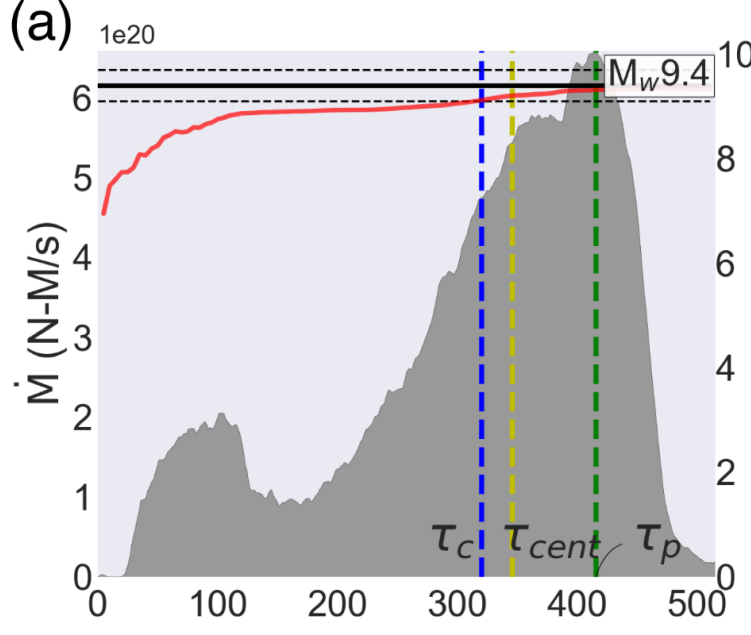

(c)

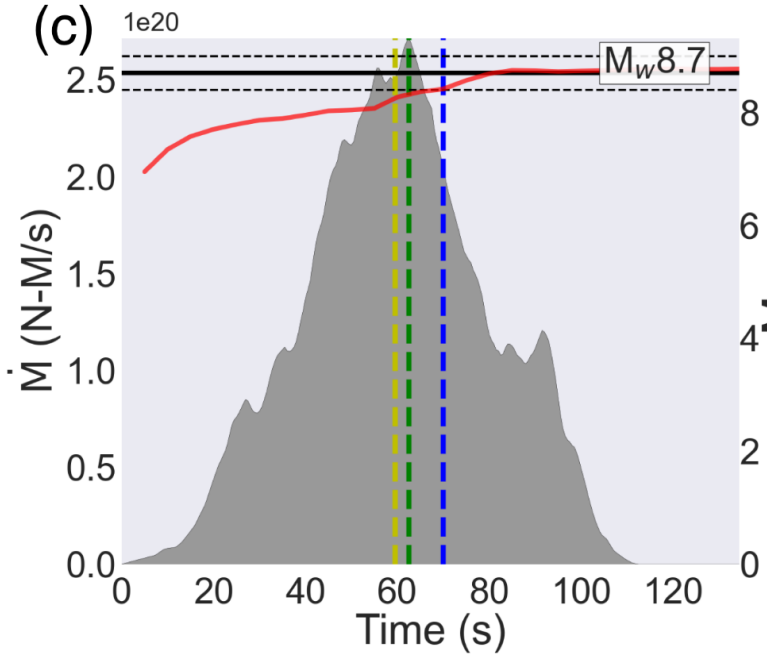

(b) $1 \mathrm{e} 20$

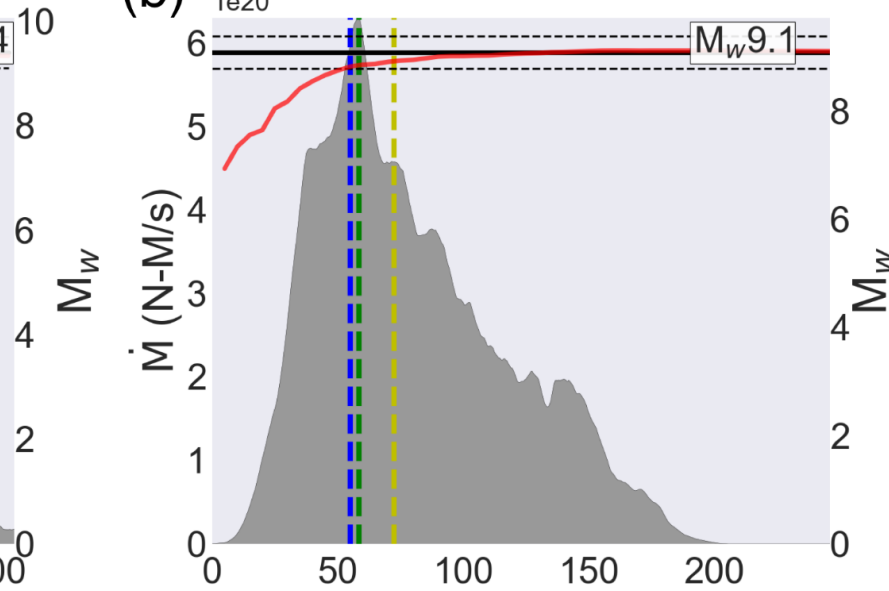

(d) 1920

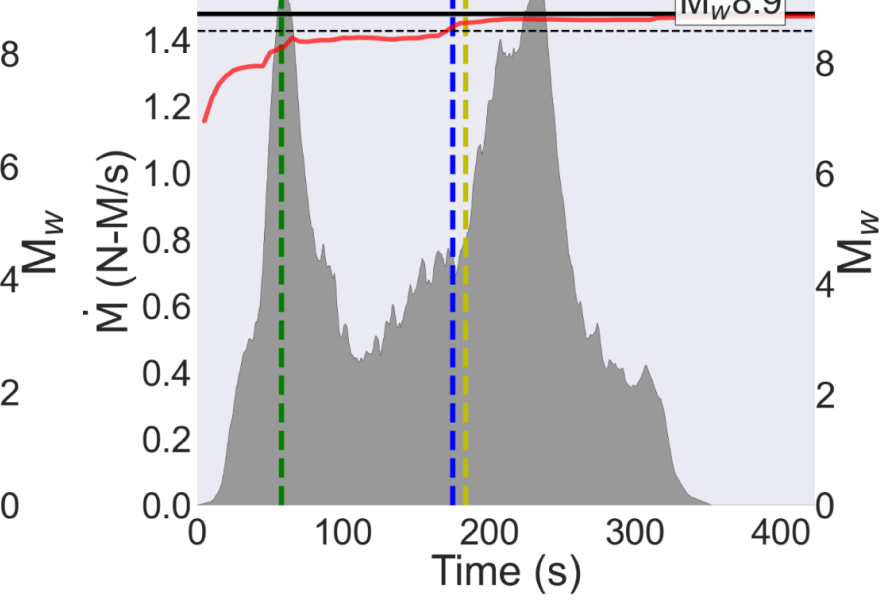

445

Figure 6. Example plot for the time to corrected prediction $\left(\tau_{c}\right)$, centroid time $\left(\tau_{\text {cent }}\right)$, and peak time $\left(\tau_{p}\right)$ for

4 different cases. Red and black lines show the M-LARGE predictions and final magnitudes, respectively.

448 Black dashed lines denote the +/-0.3 magnitude unit range. (a) shows the case of late rupturing, where the source focuses at the end of the rupture. (b) shows the case with early rupturing, where the source focuses at the beginning of the rupture. (c)

(c) nearly symmetric (triangular) source time function

(d) shows the case

451 of two rupture asperities. 
One of the challenges for developing algorithms to characterize large earthquakes is the rarity of real events. However, while large earthquakes are infrequent, their rupture kinematics

458 are not unexpected. They are the same as what we image in more modestly sized events. If a 459 comprehensive and realistic rupture scenario dataset can be generated, then for training a 460 machine learning algorithm, large earthquakes are no longer "rare". We can rely on the synthetic 461 data as a sufficient representation of the real world. To test this we compare the records of the 4622010 Mw8.8 Maule earthquake to the 25,760 scenario events in the training dataset. These 463 rupture scenarios have no a-priori knowledge of the source behavior of the Maule earthquake, 464 but simply follow the assumptions in section 2.1. In Figure S8 we use a grid-search to select 5 best fitting events from the dataset to the Maule records, and they all have similar moment magnitudes (i.e. events with similar PGD patterns have similar fault slips). This suggests that large earthquake's kinematics are not unmodeled, and given sufficient realistic simulations, rarity is no longer an issue for training.

\subsection{Timing of final magnitude estimation}

Although the timeliness of the final magnitude assessment is intimately tied to the evolution

472 of the STFs (i.e. whether the event grows faster or slower), we find that frequently the time to 473 correct predictions do not follow the exact STF behavior. The reason for this time variation is 474 mainly due to the effect of the $+/-0.3$ tolerance. A successful prediction can occur earlier than the 475 actual source duration and at the lower bound of the magnitude tolerance (e.g. Figure 6) resulting 476 in an earlier prediction. While the effect of the magnitude tolerance depends on the shape of the 477 individual STF, which is non-trivial to our stochastic simulations; however, by simply assuming 478 the STF as a triangular function (i.e. rise and fall-off rates are the same), we can estimate this 479 being $71 \%$ of the original duration time based on the scaling of Duputel et al. (2013) (a detailed 480 derivation is provided in the Text $\mathrm{S} 2$ in the supporting information). For example, on average, a 481 Mw9.0 event takes $\sim 170 \mathrm{~s}$ to rupture, while it only takes $120 \mathrm{~s}$ to rise to the acceptable Mw 
482 threshold of Mw8.7. Our model result shows an even shorter magnitude determine time, which 483 is about $25 \%-50 \%$ of the source duration (Figure $7 a$ ). This advance in time is when we consider 484 sources that follow a non-symmetric flat and long tail Dreger-STF, which have growth patterns 485 that frequently be seen in worldwide databases (Figure 7b) (Mena et al., 2010). Thus, our model 486 can provide practical earlier warning while updating its magnitude as time progresses; this is only 487 possible when the real-time STF can be accurately measured.

(a)

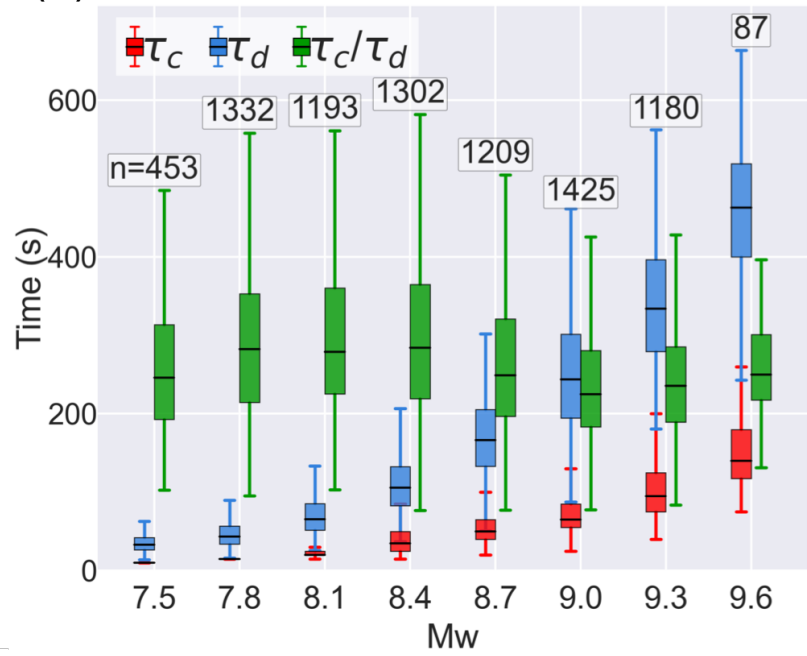

(b) 1821

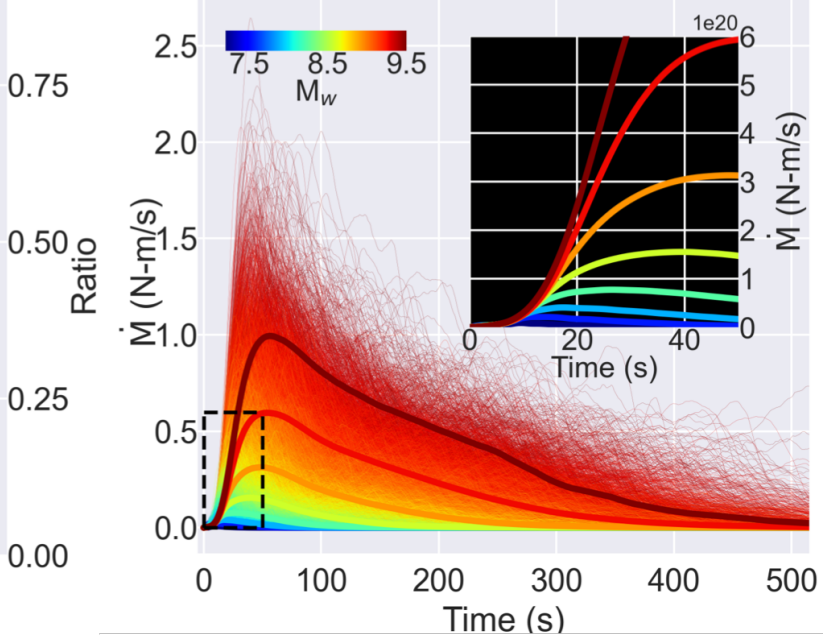

492 Figure 7. Warning time ratios and STF analysis. (a) shows the duration $\left(\tau_{d}\right)$, time to the correct prediction $493\left(\tau_{c}\right)$, and the ratio between these two for each magnitude bin. Texts indicate the number of samples for 494 each bin. (b) shows the STF of 36800 rupture scenarios color coded by Mw. Thick lines denote the averaged 495 STF of different magnitude bins. Inset shows the zoom-in view of the averaged source time functions. 
The uncertainty in magnitude prediction is important for practical EEW systems. A

500 probabilistic output layer could potentially be an estimator of the model confidence; however, in

501 our regression-type model, such an output layer is not straightforward. Here, we analyze the

502 model performance on the testing dataset to estimate uncertainty. Assuming that the distribution

503 of the testing dataset is complete, we calculate the model accuracy as a function of time (i.e.

504 length of PGD data used) and its magnitude (Figure 8). Figure 8a shows the prediction accuracies

505 with respect to their final magnitudes. We find that generally high accuracies occurred at the right-

506 hand side of the estimated duration curve, suggesting a final magnitude is more likely to be

507 correctly determined after the source termination. For example, the predicted magnitude of Mw9.0

508 has an accuracy of $77 \%$ at $100 \mathrm{~s}$, and this rises to $98 \%$ when estimating the same magnitude at

$509200 \mathrm{~s}$. On the other hand, the low accuracies at the beginning of the prediction suggests that the

510 initial rupture signals are not good indicators of final magnitude, which is consistent with previous

511 source studies (Rydelek \& Horiuchi, 2006; Meier et al., 2017; Goldberg et al., 2018; Ide, 2019;

512 Melgar \& Hayes, 2019). This is also demonstrated in Figure S9, where the same current STF

513 shapes may lead to different final magnitudes. We also note that for very large events (Mw9.2+),

514 high accuracies can occur at the very early stage, prior to the source duration. This is due to large

515 slip influencing the beginning of the STF (e.g. Figure 6b), and since the largest possible magnitude

516 is limited by the finite fault geometry (i.e. in our case, Mw9.6), the possibility that a Mw9.2+ event

517 grows into a larger event is limited.

518

We further show the model accuracies with respect to their current/time dependent 520 magnitude (Figure 8b). This is also the actual label that the model learns during the training step.

521 However, Figure 8b shows that the misfits are not evenly distributed, large misfits occur at the 522 beginning of the prediction. The mismatches at the beginning of the data-label pairs are mainly 523 due to the travel time delay introduced in section 2.3. In addition to this, GNSS noise can also 524 dominate the beginning of the records when only few stations have true rupture signals, 
525 contributing to large uncertainty at this time. The effect of GNSS noise can also be seen in both

526 Figure 8a and Figure 8b, where larger misfits occur constantly throughout the time window for 527 smaller events (e.g. Mw below 8.5).

528

(a)
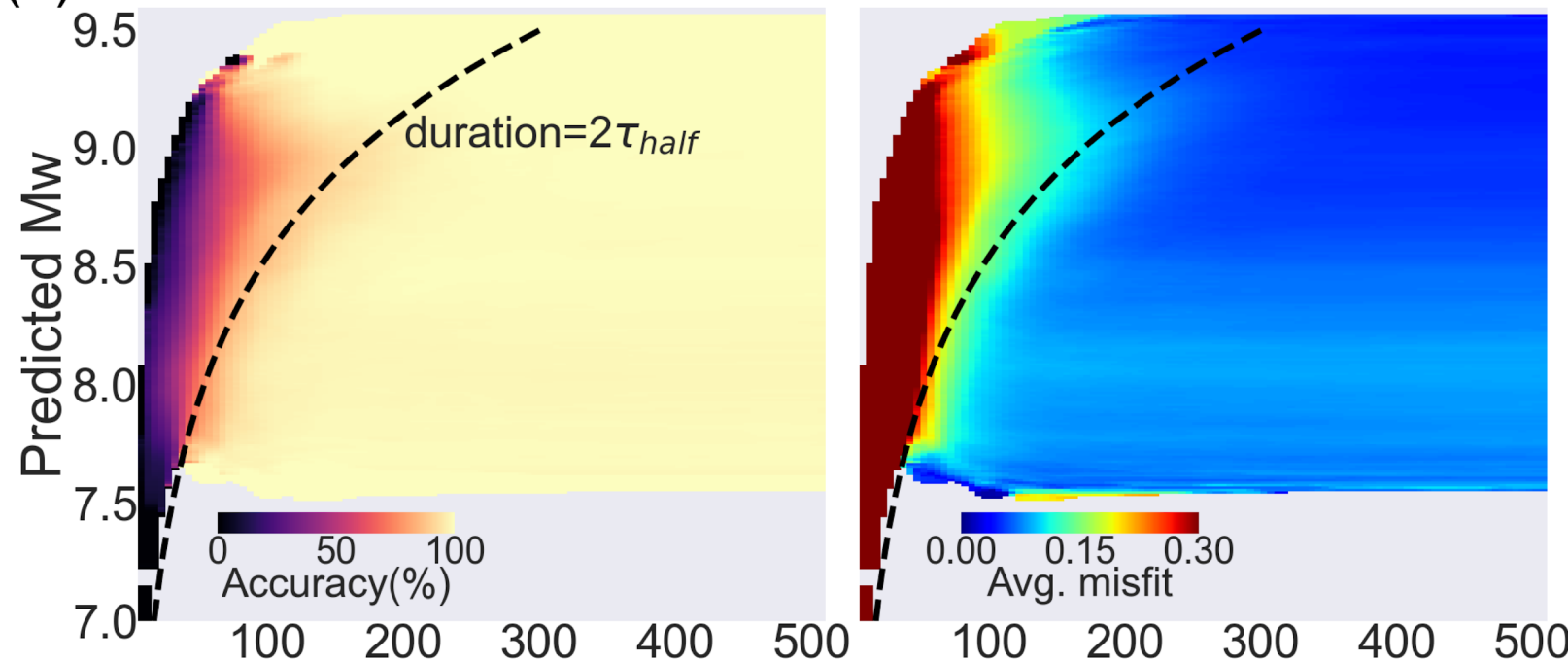

(b)
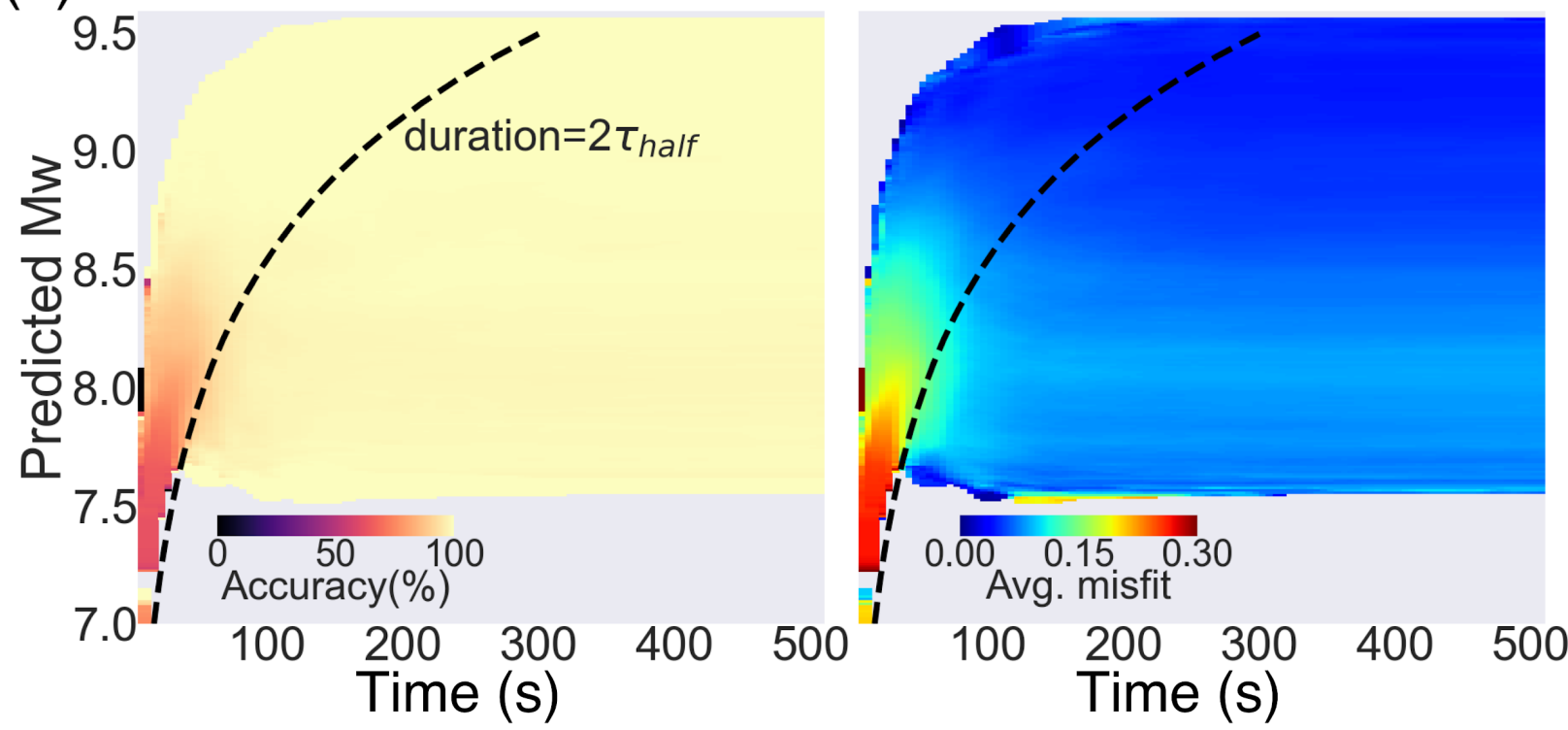

530 Figure 8. Model performance on the testing dataset. (a) shows the prediction accuracy (i.e. number of 531 success prediction/total samples) as a function of PGD time and Mw. Where a success prediction is defined 532 as when the predicted and final Mw misfit is smaller than 0.3. Dashed line shows the estimated duration from Duputel et al. (2013). (b) same as (a), but define a success prediction is when the predicted and time 
dependent Mw misfit is smaller than 0.3. Note that the time dependent Mw is the integration from the STF at current time.

\subsection{Limitations and future work}

We have shown that M-LARGE has the ability to learn complex rupture patterns from the crustal deformation data. Also, it significantly outperforms other HR-GNSS EEW algorithms.

541 However, we note that it still has some limitations, and these should be targets for potential 542 improvements in the future. First, once M-LARGE is trained, the model is not global in scope, it 543 is presently limited by the simulated earthquakes, waveforms and network geometry for a specific region. So at present, the model can only be applied to the specific configuration of GNSS sites at a specific subduction zone, Chile. In machine learning, whether a model can be applied to other data not seen in training, such as that from a different subduction zone, is called generalization.

547 It is evident that the approach we have followed here is tied to the specific network geometry and 548 subduction zone and will not immediately generalize to other tectonic settings. For M-LARGE to 549 be useful elsewhere it will need to be re-trained to another specific geometry and perceived 550 possible set of ruptures for that tectonic environment. For example, consider the Cascadia 551 Subduction Zone (CSZ) where an operational EEW system exists (Kohler et al., 2020) and 552 already uses simple PGD scaling from GNSS for magnitude calculation. Steps to implement M553 LARGE in this new setting would involve taking the known 3D geometry of the megathrust and 554 simulating an adequate set of ruptures in a desired magnitude range ( M7-M9.2), waveforms 555 would be synthesized for the 1000 GNSS station network (Murray et al., 2018), polluted with real 556 recorded GNSS real-time noise and used to train another version of M-LARGE. For such a real557 world implementation careful thought would have to go into other non-subduction zone sources 558 common to the region, such as offshore strike slip faults and inslab events. These kinds of events 559 could be simulated as well and included in training. Validation, would be challenging given the 
560 dearth of large megathrust ruptures but could be done on other eal-recorded events such as M7

561 events in the Mendocino triple junction or the M6.7 Nisqually earthquake as shown by Crowell et

562 al. (2016). This discussion shows how it is non-trivial to apply the method to a new region so

563 future avenues of research will include whether the model can perhaps be generalized by other

564 means such as introducing feature engineering (e.g. extracting the hypocentral distance used by

565 GFAST). We have not yet attempted this because regional heterogeneity such as site effects,

566 tectonic environments, fault geometries, and station distribution vary, so global model

567 generalization is non-trivial. However, synthesis of the ruptures and GNSS waveforms is fast enough that the algorithm could be adapted to other environments.

M-LARGE, like any geodesy-based technique, is only useful for large magnitude events

571 typically in the M7+ range (e.g. Crowell et al., 2013; Melgar et al., 2015). Damaging shaking during

572 earthquakes can also occur at significantly smaller magnitudes (e.g. Minson et al., 2021). The

573 approach proposed here is not meant to replace seismic methods but rather to work in tandem

574 with them. Saturation is a persistent concern for EEW and by combining networks, data types, 575 and algorithms EEW systems can respond to a wider variety of events.

We note that for the 2010 Mw8.8 Maule earthquake example, there is a $17 \mathrm{~s}$ gap without

578 recording due to lack of near-field stations. This performance could be sped up by $\sim 10 \mathrm{~s}$ if the

579 information delay introduced by the travel times could be reduced, i.e. if station coverage were

580 expanded offshore. The model performance is strongly reliant on the training dataset behaving

581 according to what is seen in world databases. As a result, an outlier event with a unique rupture 582 may still prove challenging. More simulated events that incorporate rupture variability would 583 improve M-LARGE's performance by making it resilient to complex rupture scenarios. 
The model architecture and hyperparameters are selected arbitrarily; however, the scale of hyperparameters are comparable to those in similar studies (e.g. Ross et al., 2018; Zhu \& Beroza, 587 2019). We do not find significant model improvement from tuning the hyperparameters we used

588 (Figure 2), probably because the model has already reached its accuracy limit (i.e. 99\%) based 589 on the current architecture. Any further improvement will require different model design. We find 590 that the logarithmic scaling function of PGD features has better performance against the 591 commonly adopted linear scaling. This is consistent with the existence of log-linear PGD and magnitude relationships (Crowell et al., 2016; Melgar et al., 2015; Ruhl et al., 2019) making the input and output pairs less complicated during model training.

We trained the model to learn the evolution of STF directly, which is based on the assumption that earthquake sources are not strongly deterministic (Rydelek \& Horiuchi, 2006; Ide, 2019), this is also the source characteristic for our simulations (Figure 7b), where initial rupture signal (i.e. < 5 s) do not provide the information of final magnitude, as opposed to strongly deterministic scenarios (Wu \& Zhao, 2006; Olson \& Allen, 2005) where the initial rupture signal for small or large magnitude events are fundamentally different. Even though the final magnitude can be made earlier than the source termination, which has been discussed in section 4.1, earthquake determinism is not the main exploration in this study. However, source observation studies with moderate or weakly deterministic behavior (Meier et al., 2017; Goldberg et al., 2018; Melgar \& 604 Hayes, 2019) may be incorporated into future models to speed up the warning time. Also, as 605 shown in Figure S9, possibility of the final magnitude can be inferred by tracking the STF's shape 606 when rupture proceed, providing another way to potentially speed up the warning time. 609 location, rupture length, width, and slip are equally important for an accurate ground motion 610 prediction or tsunami amplitude forecasts. In this paper, we have successfully demonstrated that 
611 M-LARGE is capable of learning Mw directly from raw observations. This is a starting point for

612 new types of EEW algorithm, and we anticipate that, given this success M-LARGE could be

613 expanded to directly forecast earthquake hazards.

\section{Conclusion}

617 Developing frameworks to provide timely warning during the largest magnitude earthquakes 618 remains an outstanding scientific and technological challenge. EEW systems continue to expand 619 and have proliferated to many countries across the globe (Allen \& Melgar, 2019). Despite this, 620 how these systems will perform in rare but high consequence, large magnitude earthquakes is 621 uncertain. Here, we have combined knowledge of where great earthquakes will occur, their 622 average expected rupture characteristics, state of the art sensor technology, and deep learning 623 to rapidly characterize large magnitude earthquakes from their crustal deformation patterns. The 624 resulting EEW algorithm, M-LARGE, has significantly better performance than current algorithms 625 and can readily be applied to any specific region capable of generating large events. As such, M626 LARGE represents a new approach to EEW that if made operational, will obviate many of the 627 performance limitations of current technologies providing accurate and fast alerts that will lead to 628 increased resilience.

631 Data availability

632 The rupture simulations and waveforms can be found on Zenodo: 633 https://doi.org/10.5281/zenodo.5015610 (Lin et al., 2020). The code of M-LARGE can be obtained 634 at https://doi.org/10.5281/zenodo.4527253 (Lin, 2020).

\section{Acknowledgement}


638 This work is funded by National Aeronautics and Space Administration NESSF grant 639 80NSSC18K1420 and National Science Foundation grants 1663834 and 1835661 . This work is 640 also partially funded by NASA grants 80 NSSC19K0360 and 80 NSSC19K1104. We thank the

641 Centro Sismologico Nacional of Chile for operation of the HR-GNSS network and access to their 642 data. We would also like to thank Amy Williamson and Brendan Crowell for discussions on the 643 performance of GFAST on simulated events.

\section{References}

Allen, R. M., \& Melgar, D. (2019), Earthquake early warning: Advances, scientific challenges, and societal needs. Annual Review of Earth and Planetary Sciences, 47, 361-388.

Báez, J. C., Leyton, F., Troncoso, C., del Campo, F., Bevis, M., Vigny, C., ... \& Blume, F. (2018), The Chilean GNSS network: Current status and progress toward early warning applications. Seismological Research Letters, 89(4), 1546-1554.

Blaser, L., Krüger, F., Ohrnberger, M., \& Scherbaum, F. (2010), Scaling relations of earthquake source parameter estimates with special focus on subduction environment. Bulletin of the Seismological Society of America, 100(6), 2914-2926.

Bock, Y., \& Melgar, D. (2016), Physical applications of GPS geodesy: A review. Reports on Progress in Physics, 79(10), 106801.

Boore, D. M., \& Bommer, J. J. (2005). Processing of strong-motion accelerograms: needs, options and consequences. Soil Dynamics and Earthquake Engineering, 25(2), 93-115.

Böse, M., Heaton, T. H., \& Hauksson, E. (2012), Real-time finite fault rupture detector (FinDer) for large earthquakes. Geophysical Journal International, 191(2), 803-812.

Cochran, E. S., Bunn, J., Minson, S. E., Baltay, A. S., Kilb, D. L., Kodera, Y., \& Hoshiba, M. (2019), Event Detection Performance of the PLUM Earthquake Early Warning Algorithm in Southern CaliforniaEvent Detection Performance of the PLUM Earthquake Early Warning Algorithm in Southern California. Bulletin of the Seismological Society of America, 109(4), 15241541.

Colombelli, S., Allen, R. M., \& Zollo, A. (2013), Application of real-time GPS to earthquake early warning in subduction and strike-slip environments. Journal of Geophysical Research: Solid Earth, 118(7), 3448-3461.

Crowell, B. W., Melgar, D., Bock, Y., Haase, J. S., \& Geng, J. (2013), Earthquake magnitude 679 
680 Crowell, B. W., Schmidt, D. A., Bodin, P., Vidale, J. E., Gomberg, J., Renate Hartog, J., ... \&

Jamison, D. G. (2016), Demonstration of the Cascadia G-FAST geodetic earthquake early warning system for the Nisqually, Washington, earthquake. Seismological Research Letters, 87(4), 930-943.

Crowell, B. W., Schmidt, D. A., Bodin, P., Vidale, J. E., Baker, B., Barrientos, S., \& Geng, J. (2018a). G-FAST earthquake early warning potential for great earthquakes in Chile. Seismological Research Letters, 89(2A), 542-556.

Crowell, B. W., Melgar, D., \& Geng, J. (2018b). Hypothetical real-time GNSS modeling of the 2016 M w 7.8 Kaikōura earthquake: Perspectives from ground motion and tsunami inundation prediction. Bulletin of the Seismological Society of America, 108(3B), 1736-1745.

DeMets, C., Gordon, R. G., \& Argus, D. F. (2010), Geologically current plate motions. Geophysical Journal International, 181(1), 1-80.

Duputel, Z., Tsai, V. C., Rivera, L., \& Kanamori, H. (2013). Using centroid time-delays to characterize source durations and identify earthquakes with unique characteristics. Earth and Planetary Science Letters, 374, 92-100.

Frankel, A., Wirth, E., Marafi, N., Vidale, J., \& Stephenson, W. (2018), Broadband Synthetic Seismograms for Magnitude 9 Earthquakes on the Cascadia Megathrust Based on 3D Simulations and Stochastic Synthetics, Part 1: Methodology and Overall ResultsMethodology and Overall Results. Bulletin of the Seismological Society of America, 108(5A), 2347-2369.

Geng, J., Pan, Y., Li, X., Guo, J., Liu, J., Chen, X., \& Zhang, Y. (2018). Noise characteristics of high-rate multi-GNSS for subdaily crustal deformation monitoring. Journal of Geophysical Research: Solid Earth, 123(2), 1987-2002.

Glorot, X., Bordes, A., \& Bengio, Y. (2011), Deep sparse rectifier neural networks. In Proceedings of the fourteenth international conference on artificial intelligence and statistics (pp. 315-323).

Goldberg, D. E., Melgar, D., Bock, Y., \& Allen, R. M. (2018), Geodetic observations of weak determinism in rupture evolution of large earthquakes. Journal of Geophysical Research: Solid Earth, 123(11), 9950-9962.

Goldberg, D. E., Melgar, D., \& Bock, Y. (2019), Seismogeodetic P-wave amplitude: No evidence for strong determinism. Geophysical research letters, 46(20), 11118-11126.

Goldberg, D. E., \& Melgar, D. (2020). Generation and validation of broadband synthetic P waves in semistochastic models of large earthquakes. Bulletin of the Seismological Society of America, 110(4), 1982-1995.

Grapenthin, R., Johanson, I. A., \& Allen, R. M. (2014), Operational real-time GPS-enhanced earthquake early warning. Journal of Geophysical Research: Solid Earth, 119(10), 7944-7965.

Graves, R. W., \& Pitarka, A. (2010), Broadband ground-motion simulation using a hybrid approach. Bulletin of the Seismological Society of America, 100(5A), 2095-2123.

Hayes, G. P. (2017), The finite, kinematic rupture properties of great-sized earthquakes since 1990. Earth and Planetary Science Letters, 468, 94-100. 
731

732

733

734

735

736

737

738

739

740

741

742

743

744

745

746

747

748

749

750

751

752

753

754

755

756

757

758

759

760

761

762

763

764

765

766

767

768

769

770

771

772

773

774

775

776

777

778

779

Hayes, G. P., Moore, G. L., Portner, D. E., Hearne, M., Flamme, H., Furtney, M., \& Smoczyk, G. M. (2018), Slab2, a comprehensive subduction zone geometry model. Science, 362(6410), 5861.

Hochreiter, S., \& Schmidhuber, J. (1997), Long short-term memory. Neural computation, 9(8), 1735-1780.

Hoshiba, M., Iwakiri, K., Hayashimoto, N., Shimoyama, T., Hirano, K., Yamada, Y., ... \& Kikuta, H. (2011). Outline of the 2011 off the Pacific coast of Tohoku Earthquake (M w 9.0)—Earthquake Early Warning and observed seismic intensity—. Earth, planets and space, 63(7), 547-551.

Hoshiba, M., \& Ozaki, T. (2014), Earthquake Early Warning and Tsunami Warning of the Japan Meteorological Agency, and Their Performance in the 2011 off the Pacific Coast of Tohoku Earthquake (Mw9.0). In Early warning for geological disasters (pp. 1-28). Springer, Berlin, Heidelberg.

Hutchison, A. A., Böse, M., \& Manighetti, I. (2020), Improving early estimates of large earthquake's final fault lengths and magnitudes leveraging source fault structural maturity information. Geophysical Research Letters, e2020GL087539.

Ide, S. (2019), Frequent observations of identical onsets of large and small earthquakes. Nature, 573(7772), 112-116.

Kawamoto, S., Hiyama, Y., Ohta, Y., \& Nishimura, T. (2016), First result from the GEONET realtime analysis system (REGARD): the case of the 2016 Kumamoto earthquakes. Earth, Planets and Space, 68(1), 190..

Kodera, Y., Yamada, Y., Hirano, K., Tamaribuchi, K., Adachi, S., Hayashimoto, N., ... \& Hoshiba, M. (2018), The propagation of local undamped motion (PLUM) method: A simple and robust seismic wavefield estimation approach for earthquake early warning. Bulletin of the Seismological Society of America, 108(2), 983-1003.

Kodera, Y., Hayashimoto, N., Moriwaki, K., Noguchi, K., Saito, J., Akutagawa, J., ... \& Hoshiba, M. (2020), First-Year Performance of a Nationwide Earthquake Early Warning System Using a Wavefield-Based Ground-Motion Prediction Algorithm in Japan. Seismological Research Letters, 91(2A), 826-834.

Kohler, M. D., Smith, D. E., Andrews, J., Chung, A. I., Hartog, R., Henson, I., ... \& Guiwits, S. (2020). Earthquake early warning ShakeAlert 2.0: Public rollout. Seismological Research Letters, 91(3), 17631775

Kong, Q., Trugman, D. T., Ross, Z. E., Bianco, M. J., Meade, B. J., \& Gerstoft, P. (2019). Machine learning in seismology: Turning data into insights. Seismological Research Letters, 90(1), 3-14.

Larson, K. M. (2009), GPS seismology. Journal of Geodesy, 83(3-4), 227-233.

LeCun, Y., Bengio, Y., \& Hinton, G. (2015), Deep learning. nature, 521(7553), 436-444. 
780

781

782

783

784

785

786

787

788

789

790

791

792

793

794

795

796

797

798

799

800

801

802

803

804

805

806

807

808

809

810

811

812

813

814

815

816

817

818

819

820

821

822

823

824

825

826

827

828

829

830

LeVeque, R. J., Waagan, K., González, F. I., Rim, D., \& Lin, G. (2016), Generating random earthquake events for probabilistic tsunami hazard assessment. In Global Tsunami Science: Past and Future, Volume I (pp. 3671-3692). Birkhäuser, Cham.

Lin, J. T., Chang, W. L., Melgar, D., Thomas, A., \& Chiu, C. Y. (2019), Quick determination of earthquake source parameters from GPS measurements: a study of suitability for Taiwan. Geophysical Journal International, 219(2), 1148-1162.

Lin, J. T., Melgar, D., Thomas, A., \& Searcy, J. (2020). Chilean Subduction Zone rupture scenarios and waveform data [Data set]. Zenodo. https://doi.org/10.5281/zenodo.5015610

Lin, J. T. (2021). jiunting/MLARGE: First release of MLARGE (Version v1.0.0). Zenodo. http://doi.org/10.5281/zenodo.4527253

Lomax, A., Michelini, A., \& Jozinović, D. (2019). An investigation of rapid earthquake characterization using single-station waveforms and a convolutional neural network. Seismological Research Letters, 90(2A), 517-529.

Maas, A. L., Hannun, A. Y., \& Ng, A. Y. (2013), Rectifier nonlinearities improve neural network acoustic models. In Proc. icml (Vol. 30, No. 1, p. 3).

Meier, M. A., Heaton, T., \& Clinton, J. (2016), Evidence for universal earthquake rupture initiation behavior. Geophysical Research Letters, 43(15), 7991-7996.

Meier, M. A., Ampuero, J. P., \& Heaton, T. H. (2017), The hidden simplicity of subduction megathrust earthquakes. Science, 357(6357), 1277-1281.

Melgar, D., Bock, Y., \& Crowell, B. W. (2012), Real-time centroid moment tensor determination for large earthquakes from local and regional displacement records. Geophysical Journal International, 188(2), 703-718.

Melgar, D., Crowell, B. W., Bock, Y., \& Haase, J. S. (2013), Rapid modeling of the 2011 Mw 9.0 Tohoku-Oki earthquake with seismogeodesy. Geophysical Research Letters, 40(12), 2963-2968.

Melgar, D., Crowell, B. W., Geng, J., Allen, R. M., Bock, Y., Riquelme, S., ... \& Ganas, A. (2015), Earthquake magnitude calculation without saturation from the scaling of peak ground displacement. Geophysical Research Letters, 42(13), 5197-5205.

Melgar, D., LeVeque, R. J., Dreger, D. S., \& Allen, R. M. (2016), Kinematic rupture scenarios and synthetic displacement data: An example application to the Cascadia subduction zone. Journal of Geophysical Research: Solid Earth, 121(9), 6658-6674.

Melgar, D., \& Hayes, G. P. (2017), Systematic observations of the slip pulse properties of large earthquake ruptures. Geophysical Research Letters, 44(19), 9691-9698.

Melgar, D., \& Hayes, G. P. (2019), Characterizing large earthquakes before rupture is complete. Science Advances, 5(5), eaav2032. 
Melgar, D., Crowell, B. W., Melbourne, T. I., Szeliga, W., Santillan, M., \& Scrivner, C. (2020), Noise characteristics of operational real-time high-rate GNSS positions in a large aperture network. Journal of Geophysical Research: Solid Earth, 125(7), e2019JB019197.

Mena, B., Mai, P. M., Olsen, K. B., Purvance, M. D., \& Brune, J. N. (2010). Hybrid broadband ground-motion simulation using scattering Green's functions: Application to large-magnitude events. Bulletin of the Seismological Society of America, 100(5A), 2143-2162.

Minson, S. E., Murray, J. R., Langbein, J. O., \& Gomberg, J. S. (2014), Real-time inversions for finite fault slip models and rupture geometry based on high-rate GPS data. Journal of Geophysical Research: Solid Earth, 119(4), 3201-3231.

Minson, S. E., Baltay, A. S., Cochran, E. S., McBride, S. K., \& Milner, K. R. (2021). Shaking is almost always a surprise: The earthquakes that produce significant ground motion. Seismological Society of America, 92(1), 460-468.

Mousavi, S. M., \& Beroza, G. C. (2020a), A machine-learning approach for earthquake magnitude estimation. Geophysical Research Letters, 47(1), e2019GL085976.

Mousavi, S. M., Ellsworth, W. L., Zhu, W., Chuang, L. Y., \& Beroza, G. C. (2020b), Earthquake transformer-an attentive deep-learning model for simultaneous earthquake detection and phase picking. Nature communications, 11(1), 1-12.

Murray, J. R., Crowell, B. W., Grapenthin, R., Hodgkinson, K., Langbein, J. O., Melbourne, T., ... \& Schmidt, D. A. (2018), Development of a geodetic component for the US West Coast earthquake early warning system. Seismological Research Letters, 89(6), 2322-2336.

Olson, E. L., \& Allen, R. M. (2005), The deterministic nature of earthquake rupture. Nature, 438(7065), 212-215.

Perol, T., Gharbi, M., \& Denolle, M. (2018), Convolutional neural network for earthquake detection and location. Science Advances, 4(2), e1700578.

Pitarka, A., Graves, R., Irikura, K., Miyakoshi, K., \& Rodgers, A. (2020). Kinematic rupture modeling of ground motion from the M7 Kumamoto, Japan earthquake. Pure and Applied Geophysics, 177(5), 2199-2221.

Riquelme, S., Medina, M., Bravo, F., Barrientos, S., Campos, J., \& Cisternas, A. (2018), W-phase real-time implementation and network expansion from 2012 to 2017: The experience in Chile. Seismological Research Letters, 89(6), 2237-2248.

Ross, Z. E., Meier, M. A., Hauksson, E., \& Heaton, T. H. (2018), Generalized seismic phase detection with deep learning. Bulletin of the Seismological Society of America, 108(5A), 28942901.

Ruhl, C. J., Melgar, D., Geng, J., Goldberg, D. E., Crowell, B. W., Allen, R. M., ... \& Cabral-Cano, E. (2019), A global database of strong-motion displacement GNSS recordings and an example application to PGD scaling. Seismological Research Letters, 90(1), 271-279. 
Ruiz, S., \& Madariaga, R. (2018). Historical and recent large megathrust earthquakes in Chile. Tectonophysics, 733, 37-56.

Rydelek, P., \& Horiuchi, S. (2006), Is earthquake rupture deterministic?. Nature, 442(7100), E5E6.

Srivastava, N., Hinton, G., Krizhevsky, A., Sutskever, I., \& Salakhutdinov, R. (2014), Dropout: a simple way to prevent neural networks from overfitting. The journal of machine learning research, 15(1), 1929-1958 .

Vallée, M., \& Douet, V. (2016). A new database of source time functions (STFs) extracted from the SCARDEC method. Physics of the Earth and Planetary Interiors, 257, 149-157.

van den Ende, M. P., \& Ampuero, J. P. (2020). Automated Seismic Source Characterization Using Deep Graph Neural Networks. Geophysical Research Letters, 47(17), e2020GL088690.

Williamson, A. L., Melgar, D., Crowell, B. W., Arcas, D., Melbourne, T. I., Wei, Y., \& Kwong, K. (2020), Toward near-field tsunami forecasting along the Cascadia subduction zone using rapid GNSS source models. Journal of Geophysical Research: Solid Earth, e2020JB019636.

Wu, Y. M., \& Zhao, L. (2006), Magnitude estimation using the first three seconds P-wave amplitude in earthquake early warning. Geophysical Research Letters, 33(16).

Ye, L., Lay, T., Kanamori, H., \& Rivera, L. (2016), Rupture characteristics of major and great (Mw $\geq$ 7.0) megathrust earthquakes from 1990 to 2015: 1. Source parameter scaling relationships. Journal of Geophysical Research: Solid Earth, 121(2), 826-844.

Zhu, L., \& Rivera, L. A. (2002), A note on the dynamic and static displacements from a point source in multilayered media. Geophysical Journal International, 148(3), 619-627.

Zhu, W., \& Beroza, G. C. (2019), PhaseNet: a deep-neural-network-based seismic arrival-time picking method. Geophysical Journal International, 216(1), 261-273.

\section{References From the Supporting Information}

Duputel, Z., Tsai, V. C., Rivera, L., \& Kanamori, H. (2013). Using centroid time-delays to characterize source durations and identify earthquakes with unique characteristics. Earth and Planetary Science Letters, 374, 92-100.

Goda, K., Yasuda, T., Mori, N., \& Maruyama, T. (2016), New scaling relationships of earthquake source parameters for stochastic tsunami simulation. Coastal Engineering Journal, 58(3), 1650010-1.

Graves, R. W., \& Pitarka, A. (2010), Broadband ground-motion simulation using a hybrid approach. Bulletin of the Seismological Society of America, 100(5A), 2095-2123. 
931

932

933

934

935

936

937

938

939

940

941

942

943

944

945

946

947

948

949

950

951

952

953

954

955

956

957

958

959

960

961

962

963

964

965

966

967

968

969

970

971

972

973

974

975

976

977

978
Graves, R., \& Pitarka, A. (2015), Refinements to the Graves and Pitarka (2010) broadband ground-motion simulation method. Seismological Research Letters, 86(1), 75-80.

LeVeque, R. J., Waagan, K., González, F. I., Rim, D., \& Lin, G. (2016), Generating random earthquake events for probabilistic tsunami hazard assessment. In Global Tsunami Science: Past and Future, Volume I (pp. 3671-3692). Birkhäuser, Cham.

Mai, P. M., \& Beroza, G. C. (2002), A spatial random field model to characterize complexity in earthquake slip. Journal of Geophysical Research: Solid Earth, 107(B11), ESE-10.

Melgar, D., LeVeque, R. J., Dreger, D. S., \& Allen, R. M. (2016), Kinematic rupture scenarios and synthetic displacement data: An example application to the Cascadia subduction zone. Journal of Geophysical Research: Solid Earth, 121(9), 6658-6674.

Melgar, D., \& Hayes, G. P. (2017), Systematic observations of the slip pulse properties of large earthquake ruptures. Geophysical Research Letters, 44(19), 9691-9698.

Melgar, D., \& Hayes, G. P. (2019), The correlation lengths and hypocentral positions of great earthquakes. Bulletin of the Seismological Society of America, 109(6), 2582-2593.

Mena, B., Mai, P. M., Olsen, K. B., Purvance, M. D., \& Brune, J. N. (2010), Hybrid broadband ground-motion simulation using scattering Green's functions: Application to large-magnitude events. Bulletin of the Seismological Society of America, 100(5A), 2143-2162.

Pasyanos, M. E., Masters, T. G., Laske, G., \& Ma, Z. (2014), LITHO1. 0: An updated crust and lithospheric model of the Earth. Journal of Geophysical Research: Solid Earth, 119(3), 2153-2173.

Zhu, L., \& Rivera, L. A. (2002), A note on the dynamic and static displacements from a point source in multilayered media. Geophysical Journal International, 148(3), 619-627. 
979

Corresponding author: Jiun-Ting Lin (jiunting@uoregon.edu)

\section{Contents of this file}

$988 \quad$ Text S1, S2

$989 \quad$ Figure S1 to S9

$990 \quad$ Table S1

Introduction

This supporting information includes details of the rupture scenarios and synthetic waveforms (Text S1), details of the estimated rupture duration (Test S2), 7 figures, and details of the model parameters (Table S1) supporting the main text.

\section{Text S1. Details on the rupture scenarios and synthetic waveforms}

Underpinning the $\mathrm{KL}$ expansion method is the notion that slip on a fault can be modeled as a spatially random field (Mai \& Beroza, 2002). Once a correlation function is defined then random draws can be made to obtain a stochastic slip pattern. By comparison to slip inversions from earthquakes worldwide several studies have noted that slip is best modeled by the Von Karman 
1004 correlation function (Mai \& Beroza, 2002; Goda et al., 2016; Melgar \& Hayes, 2019) where the 1005 correlation between the $i$-th and $j$-th subfault in the rupture is defined as

1006

1007

$$
C_{i j}\left(r_{i j}\right)=\frac{G_{H}\left(r_{i j}\right)}{G_{0}\left(r_{i j}\right)}
$$

1008

1009

$$
G_{H}\left(r_{i j}\right)=r_{i j}^{H} K_{H}\left(r_{i j}\right)
$$

1010

1011 where $K_{H}$ is the modified Bessel function of the second kind and $\mathrm{H}$ is the Hurst exponent. We set $1012 H=0.4$ based on a recent analysis of large earthquakes between 1990 and 2019 (Melgar \& 1013 Hayes, 2019), which is slightly lower than the value of $\mathrm{H}=0.7$ proposed when stochastic slip 1014 models were first employed (Mai \& Beroza, 2002; Graves \& Pitarka, 2010). $r_{i j}$ is the inter-subfault 1015 distance given by

1016

$$
r_{i j}=\sqrt{\left(r_{s} / a_{s}\right)^{2}+\left(r_{d} / a_{d}\right)^{2}}
$$

1018

1019

Where $r_{s}$ is the along-strike distance and $r_{d}$ the along-dip distance. The along-strike and 1020 along-dip correlation lengths, $a_{s}$ and $a_{d}$, control the predominant asperity size in the resulting slip 1021 pattern (Mai \& Beroza, 2002) and scale with indirectly with magnitude as a function of the fault 1022 length and width according to 1023

$$
\begin{aligned}
& a_{s}=2.0+\frac{1}{3} L, \\
& a_{d}=1.0+\frac{1}{3} W,
\end{aligned}
$$


Once all the parameters of the correlation matrix are defined the covariance matrix is 1028 obtained by

$$
\widehat{C_{l j}}=\sigma_{i} C_{i j} \sigma_{j}
$$

Where $\sigma$ is the standard deviation of slip which is usually defined as a fraction of mean slip. Here we set it to 0.9 (LeVeque et al., 2016). Now we can obtain a randomly generated slip pattern with the statistics as defined above by summing the eigenvectors of the covariance matrix according to the K-L expansion (LeVeque et al., 2016) such that

$$
s=\mu+\sum_{k=1}^{N} \quad z_{k} \sqrt{\lambda_{k}} v_{k}
$$

where $s$ is a column vector containing the values of slip at each of the subfaults for a particular realization. $\mu$ is the expected mean slip pattern, we set it to be a vector with enough homogenous slip over the selected subfaults to match the target magnitude. $N$ is the maximum number of 1041 summed eigenvectors. We use a reasonably large number of 100 which should give enough 1042 variation of slip complexity (Melgar et al., 2016; LeVeque et al., 2016). $z_{k}$ is a scalar randomly 1043 selected from a presumed gaussian distribution with zero mean and standard deviation of 1. $\lambda_{k}$ 1044 and $v_{k}$ denotes the eigenvalue and eigenvector of the covariance matrix.

With the stochastic slip pattern in hand, the second step is to define the rupture kinematics.

1047 Here we follow common best practices and a full treatment of this can be found in Graves \& 1048 Pitarka $(2010,2015)$. We set the rupture speed to 0.8 of the local shear wave velocity at the 1049 subfault depth plus some stochastic perturbation to destroy perfectly circular rupture fronts. The 1050 hypocenter is randomly selected from the subfaults that are involved in the rupture to ensure both 1051 unilateral and bilateral ruptures. Rise times are defined to be proportional to the square root of 
1052 local slip (Mena et al., 2010) but over the entire fault model must on average obey known rise1053 time magnitude scaling laws (Melgar \& Hayes, 2017). We then use the Dreger slip rate function 1054 to describe the time-evolution at a particular subfault (Mena et al., 2010; Melgar et al., 2016). It is 1055 well-known that the shallow megathrust has slow rupture speeds and long rise times, so for 1056 subfaults shallower than $10 \mathrm{~km}$ rupture speeds are set to 0.6 of shear wave speed and rise times 1057 are doubled from what is predicted by the scaling by the square root of slip. Below $15 \mathrm{~km}$ the 1058 previously described rules are used, and between 10 and $15 \mathrm{~km}$ depth a linear transition between 1059 the two behaviors is employed. This is similar to what is done for continental strike-slip faults 1060 (Graves \& Pitarka, 2010). Similarly, the rake vector is set to 90 degrees plus some stochastic 1061 perturbations.

1062

Once the slip pattern and its complete time evolution are known, synthetic GNSS waveforms 1064 are generated by summing all the synthetic data from participating subfaults. We use the FK 1065 package, which is a 1D frequency-wavenumber approach (Zhu \& Rivera, 2002) and the LITHO1.0 velocity structure (Pasyanos et al., 2014) to generate the Green's functions from all subfaults to 1067 given stations. We focus only on the long period displacement waveforms $(<0.5 \mathrm{~Hz}$ or 1 second 1068 sampling) since they are less sensitive to small scale crustal structure and are the dominant period 1069 of large earthquakes.

1070

1071

$$
T=2.4 \times 10^{-8} \times M_{0}^{1 / 3}
$$




$$
M_{0}=10^{(M w+10.7) \times 1.5},
$$

1079

1080 Where the $M_{0}$ represents moment in dyne-cm. By plugging the equation (9) into (8) with an 1081 magnitude $\mathrm{Mw}-0.3$, we can estimate the duration ratio

1082

1083

$$
R=\left(10^{(M w-0.3+10.7) \times 1.5} / 10^{(M w+10.7) \times 1.5}\right)^{1 / 3}=71 \%
$$

1084

1085 Thus, the duration of Mw- 0.3 can be estimated by $71 \%$ of the original Mw duration.

1086

1087

1088

1089

1090

1091

1092

1093

1094

1095 

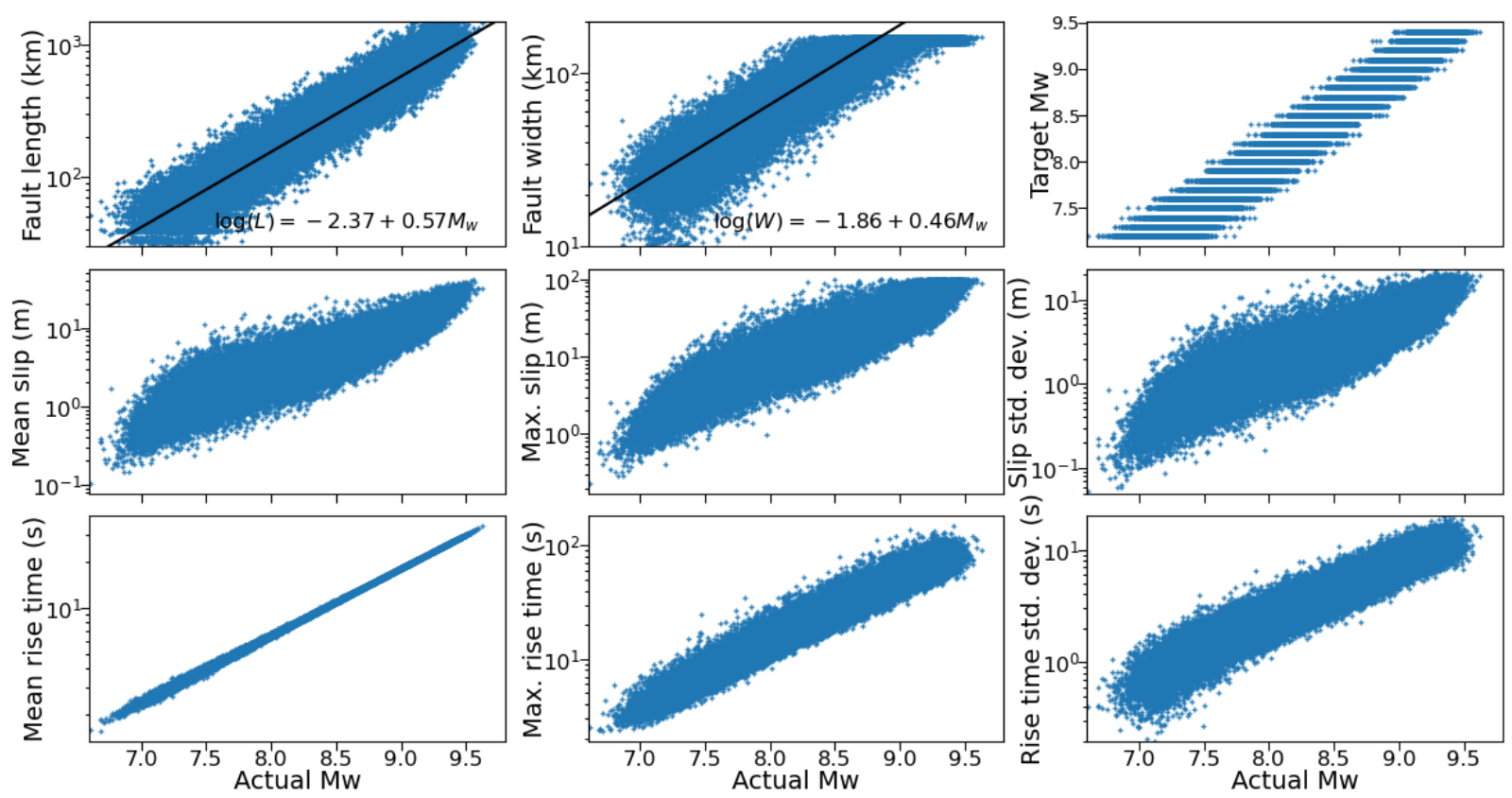

1096

Figure S1. Source parameters of the 36800 rupture scenarios.

1098

1099

1100

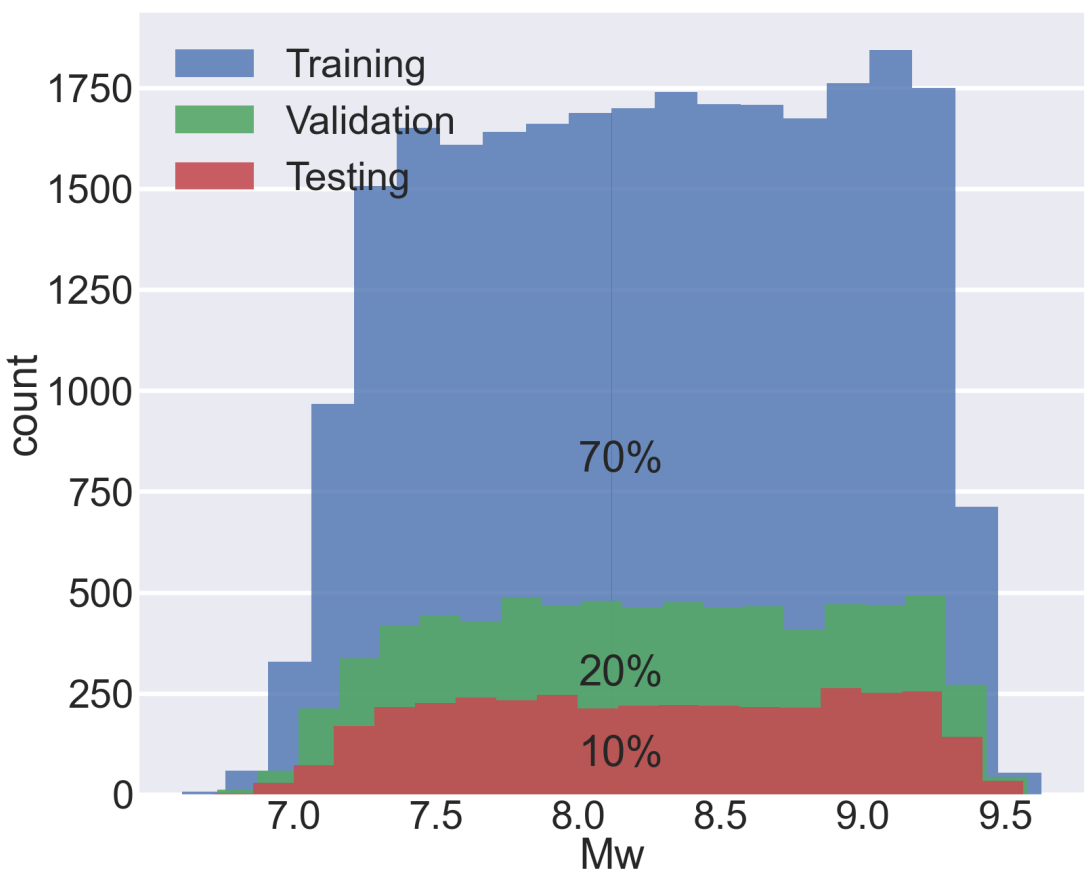

1102 Figure S2. Histogram and partition of training, validation and testing dataset. 
(a)

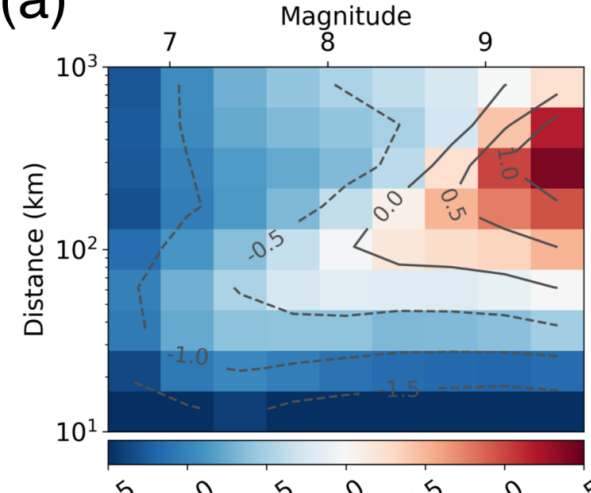

(b)

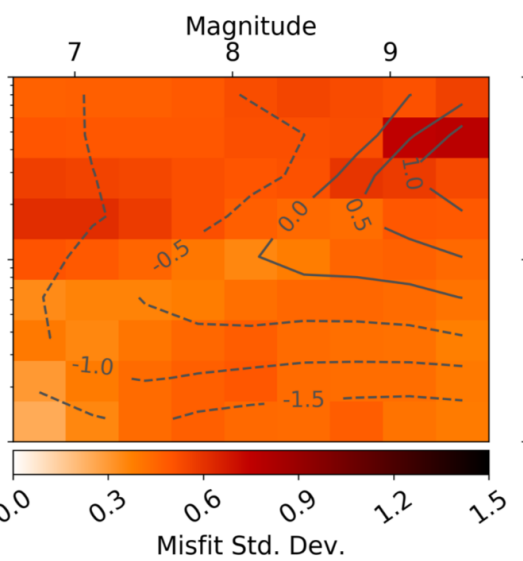
Misfit Std. Dev.
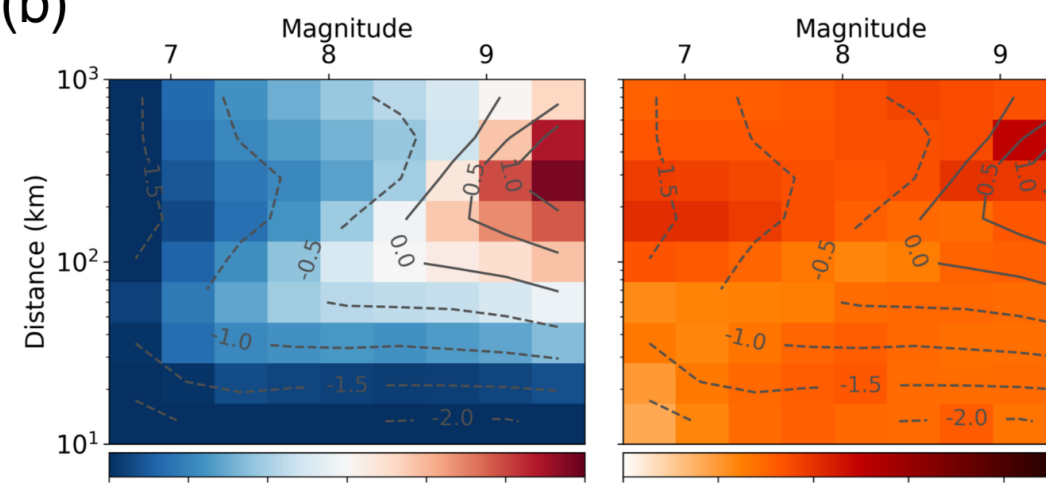

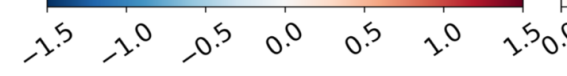
Ln(Simulation / GMPE)

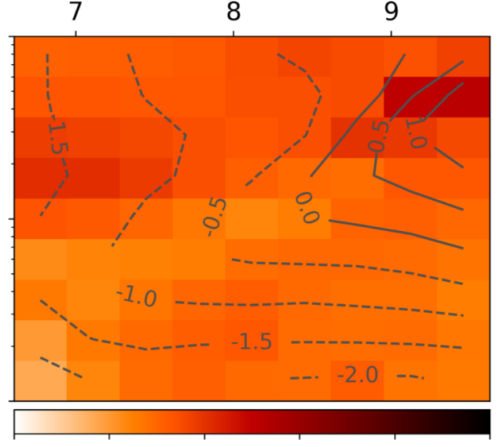

Figure S3. Comparison between synthetic data and PGD-Mw scaling of (a) Melgar et al. (2015) and (b) Ruhl et al. (2019). . (a) and (b) (b) from left to right shows the misfit of synthetic PGD and PGD-Mw scaling and its contour; standard deviation of the misfit; and distribution of waveforms in count. 
(a)

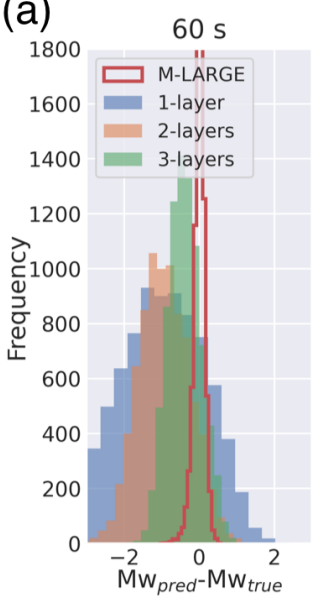

$120 \mathrm{~s}$

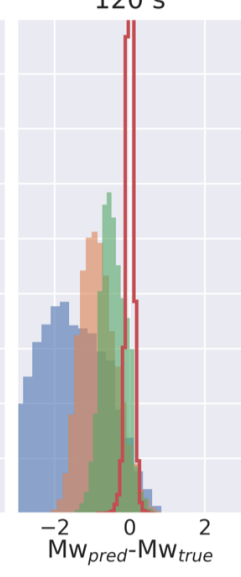

$360 \mathrm{~s}$

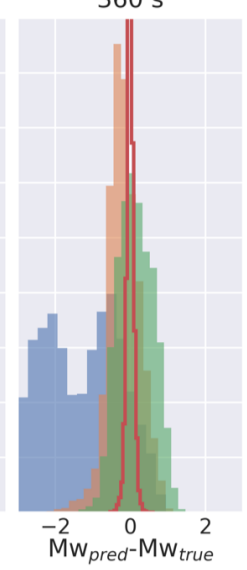

(b)

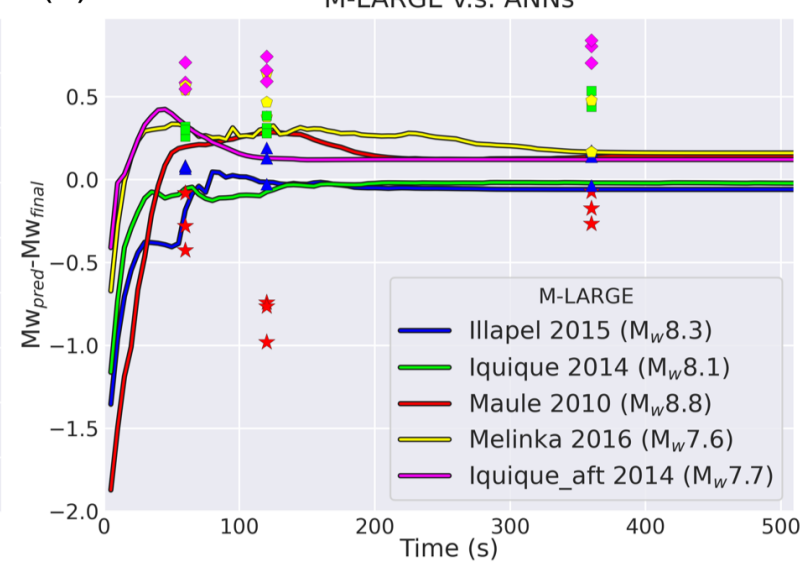

1111 Figure S4. Comparison between multi-layers artificial neural networks (ANNs) and M-LARGE (i.e.

1112 ANNs+LSTM). (a) Histograms of the Mw misfits for testing data at 60, 120, and 360 s, respectively. Each

1113 layer of ANNs model has 8 neurons and connects to a ReLU activation function. (b) Model performance on

1114 real data. Colored-lines represent the M-LARGE predictions, colored-symbols denote the predictions from

11153 ANNs models at 60, 120, 360 seconds. The figure shows that all the ANNs models have larger predicted

1116 errors than the M-LARGE. 


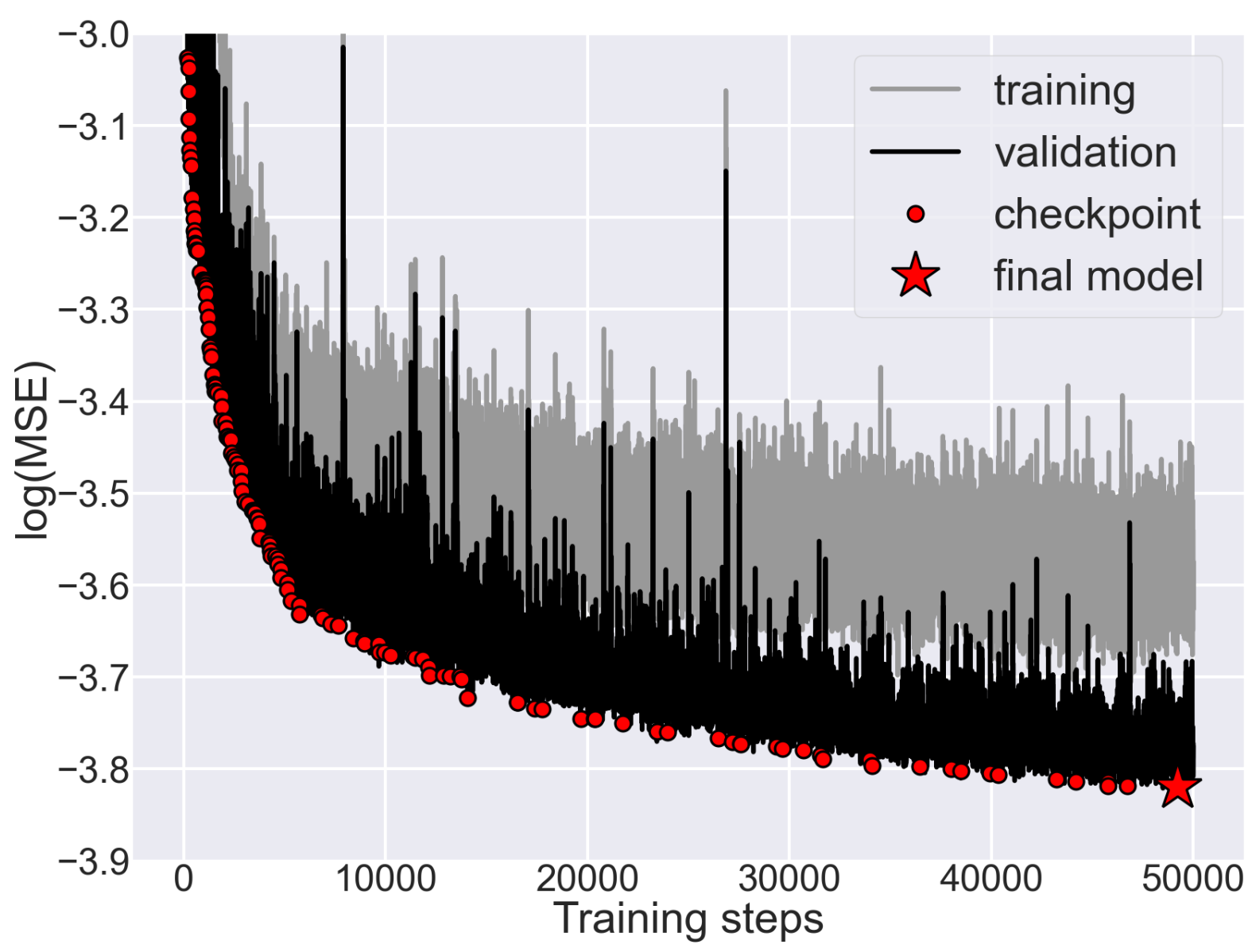

Figure S5. Training curve for M-LARGE. Light and dark line show the MSE for training and validation data, respectively. Red dots denote the checkpoints for the training, with interval of every 5 epochs and save the model if the current checkpoint loss is smaller than the previous checkpoint loss. Red star represents the

1124 final selected model, which has the minimum checkpoint loss. Note that the validation loss is smaller than the training loss because dropouts are only implemented in the loss calculation. 

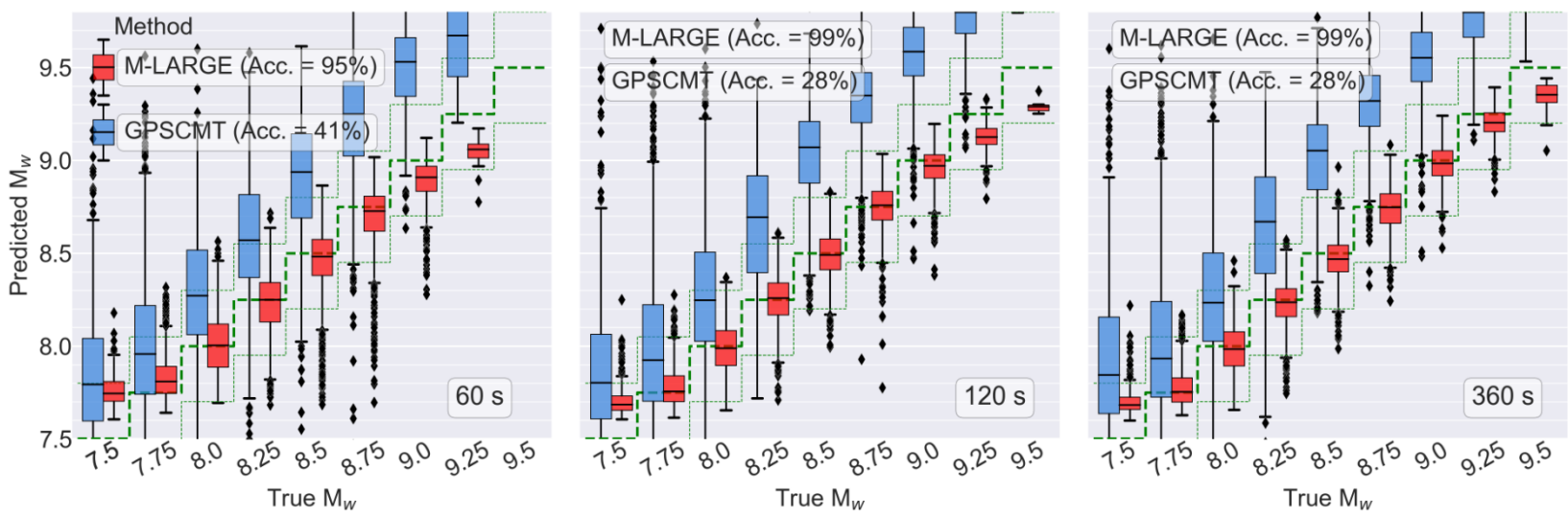

1140 Figure S6. Similar to the Figure 3b in the main text, but the comparison of the M-LARGE (red) and GPSCMT

1141 (blue) predicted magnitudes at 60,120 and $360 \mathrm{~s}$ for different magnitude bins. Model accuracies at 60, 120

1142 and $360 \mathrm{~s}$ are shown in text. The thick and thin green dashed lines show the 1:1 and \pm 0.3 reference for 1143 each magnitude bin, respectively. 


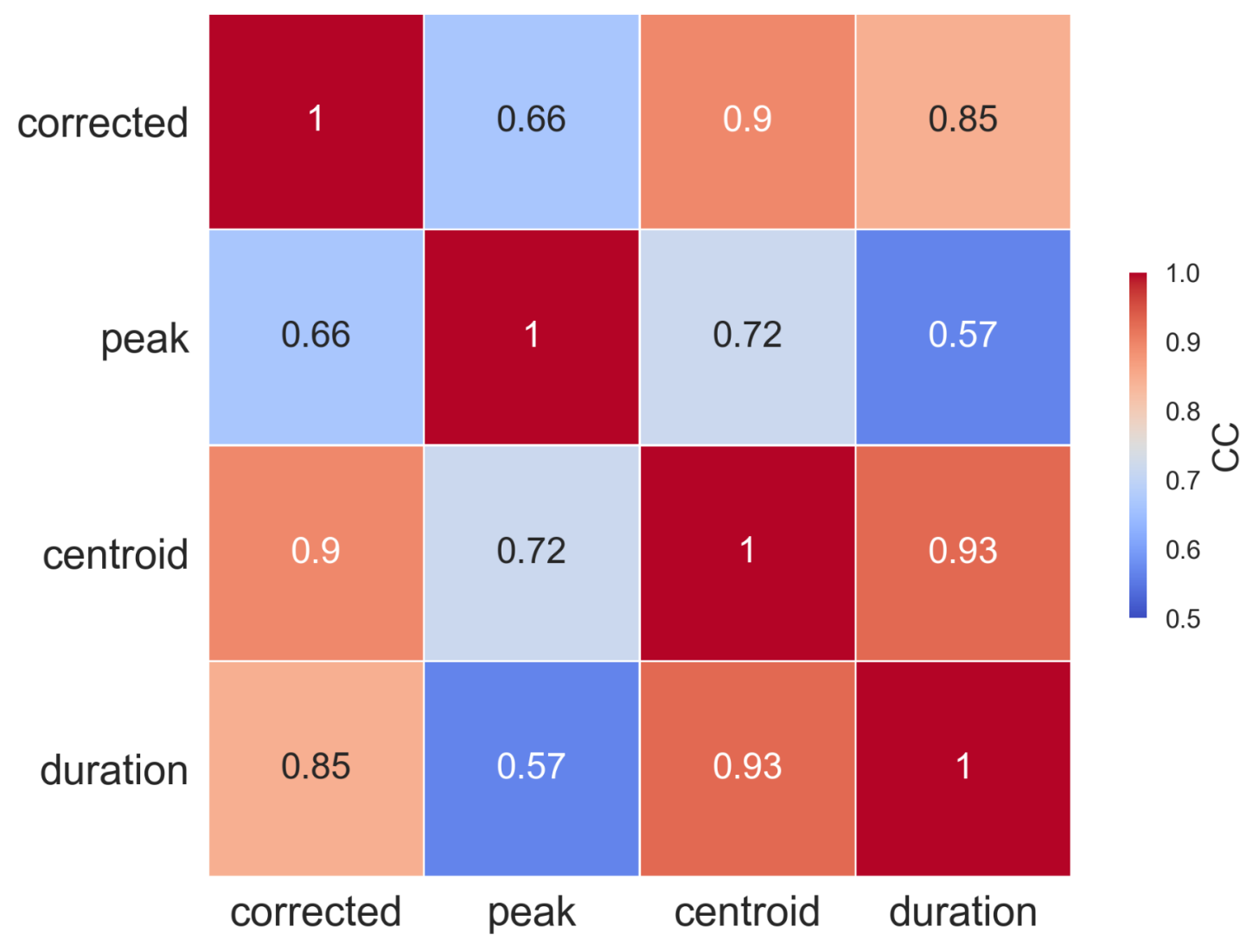

1147 Figure S7. Correlation coefficient between time to corrected prediction, peak time, controid time and the 1148 duration introduced in session 3.4 in the main text.

(a)

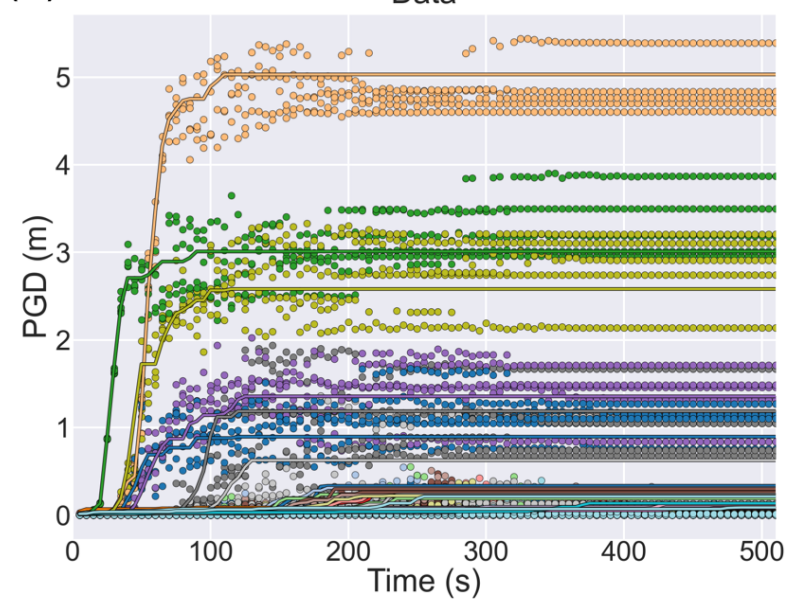

(b)

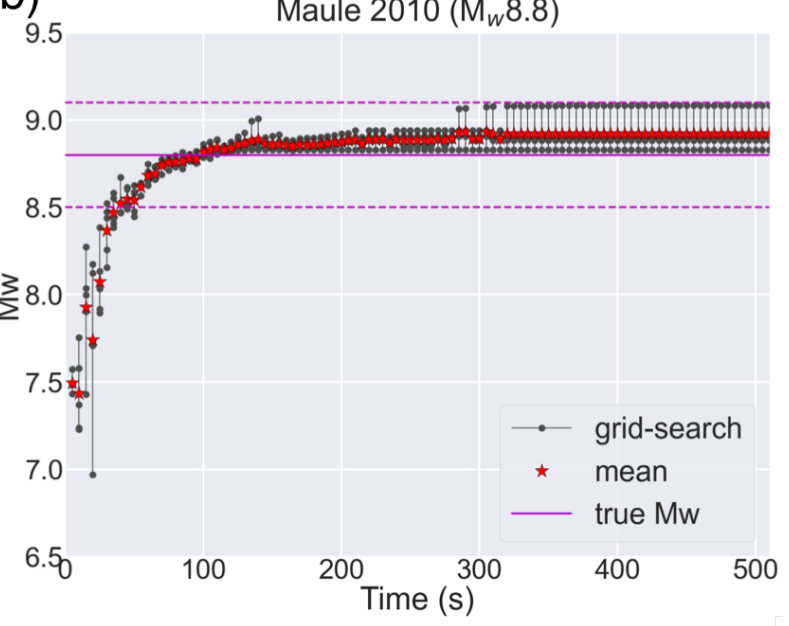


1152 Figure S8. Example of direct grid-searching from the training dataset. (a) Solid lines show the real PGD

1153

1154

1155

1156

1157

(a)

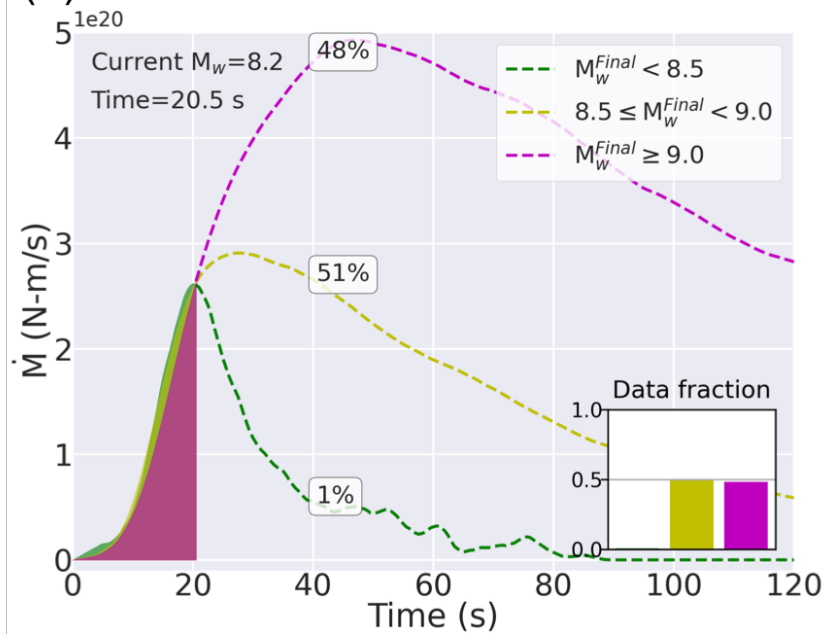
show the true $M w$ and \pm 0.3 magnitude unit, respectively. (b)

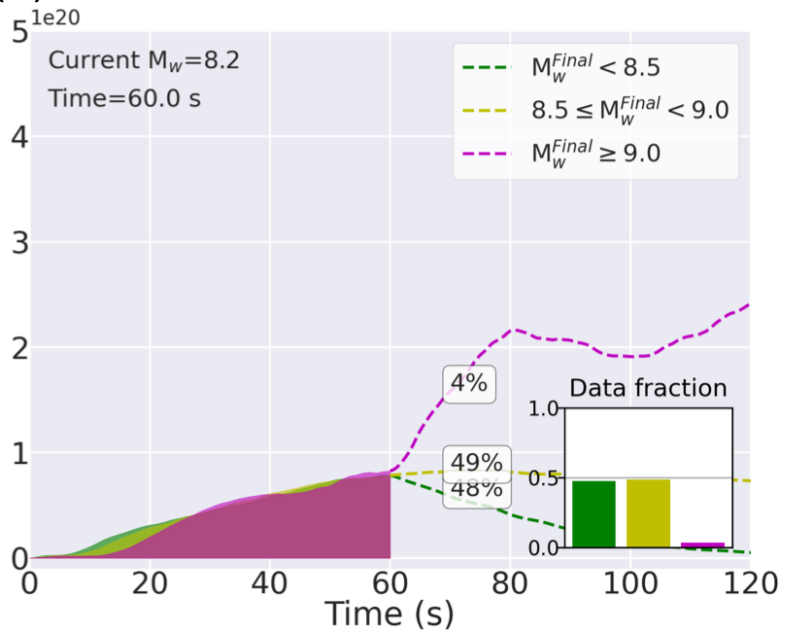

1159

1160

1161

1162

1163

1164

1165

1166

1167

1168

1169

1170

1171 data of the Maule 2010 earthquake. The dots denote the 5 best fitting PGD data for all the stations at the particular time. (b) shows the 5 magnitudes corresponding to the PGD in (a). Black dots and red stars represent the 5 magnitudes and average of the 5 magnitudes, respectively. Magenta line and dashed lines

Figure S9. Example STFs in our dataset grouped by the same current magnitude. The figure shows the sources are not strongly deterministic; however, the exact current STF can be further used to infer the possibility of final magnitude. (a) The STFs at 20.5 s have similar shapes and accumulated Mw of 8.2, however, are ambiguous to their final magnitude. The percentage texts denote the fractions of data that eventually grow to the designated groups. Dashed lines show the averaged future STFs for each group. (b) same as (a) but show the STFs at 60 s. The statistic shows it is less likely (i.e. 4\%) that an event can grow to a very large event although some large Mw earthquakes take hundreds of seconds to rupture. The possibility is restricted according to the current rupture history and the remaining available space of growth limited by the subduction zone geometry. 
1175 Table S1. List of parameter values used

\begin{tabular}{|l|l|l|l|l|l|}
\hline Layer\# & Name & Size & Input dimension & Output dimension & Trainable parameters \\
\hline Layer0 & Input & & {$[N, 102,242]$} & {$[N, 102,242]$} & \\
\hline Layer1 & Dense & 256 & {$[N, 102,242]$} & {$[N, 102,256]$} & 62208 \\
\hline Activation & LeakyReLU & 0.1 & {$[N, 102,256]$} & {$[N, 102,256]$} & \\
\hline Layer2 & Dense & 256 & {$[N, 102,256]$} & {$[N, 102,256]$} & 65792 \\
\hline Activation & LeakyReLU & 0.1 & {$[N, 102,256]$} & {$[N, 102,256]$} & \\
\hline Layer3 & Dropout & 0.2 & {$[N, 102,256]$} & {$[N, 102,256]$} & \\
\hline Recurrent input & & & & & \\
\hline Layer4 & LSTM & 128 & {$[N, 102,256]$} & {$[N, 102,128]$} & 197120 \\
\hline Layer5 & Dense & 128 & {$[N, 102,128]$} & {$[N, 102,128]$} & 16512 \\
\hline Activation & LeakyReLU & 0.1 & {$[N, 102,128]$} & {$[N, 102,128]$} & \\
\hline Layer6 & Dense & 64 & {$[N, 102,128]$} & {$[N, 102,64]$} & 8256 \\
\hline Activation & LeakyReLU & 0.1 & {$[N, 102,64]$} & {$[N, 102,64]$} & \\
\hline Layer7 & Dense & 32 & {$[N, 102,64]$} & {$[N, 102,32]$} & 2080 \\
\hline Activation & LeakyReLU & 0.1 & {$[N, 102,32]$} & {$[N, 102,32]$} & \\
\hline Layer8 & Dense & 8 & {$[N, 102,32]$} & {$[N, 102,8]$} & 264 \\
\hline Activation & LeakyReLU & 0.1 & {$[N, 102,8]$} & {$[N, 102,8]$} & \\
\hline Layer9 & Dropout & 0.2 & {$[N, 102,8]$} & {$[N, 102,8]$} & \\
\hline Layer10 & Dense & 1 & {$[N, 102,8]$} & {$[N, 102,1]$} & 9 \\
\hline
\end{tabular}




\section{References From the Supporting Information}

1182

1183

1184

1185

1186

1187

1188

1189

1190

1191

1192

1193

1194

1195

1196

1197

1198

1199

1200

1201

1202

1203

1204

1205

1206

1207

1208

1209

1210

1211

1212

1213

1214

1215

1216

1217

1218

1219

1220

1221

1222

1223

1224

1225

1226

1227

Duputel, Z., Tsai, V. C., Rivera, L., \& Kanamori, H. (2013). Using centroid time-delays to characterize source durations and identify earthquakes with unique characteristics. Earth and Planetary Science Letters, 374, 92-100.

Goda, K., Yasuda, T., Mori, N., \& Maruyama, T. (2016), New scaling relationships of earthquake source parameters for stochastic tsunami simulation. Coastal Engineering Journal, 58(3), 1650010-1.

Graves, R. W., \& Pitarka, A. (2010), Broadband ground-motion simulation using a hybrid approach. Bulletin of the Seismological Society of America, 100(5A), 2095-2123.

Graves, R., \& Pitarka, A. (2015), Refinements to the Graves and Pitarka (2010) broadband ground-motion simulation method. Seismological Research Letters, 86(1), 75-80.

LeVeque, R. J., Waagan, K., González, F. I., Rim, D., \& Lin, G. (2016), Generating random earthquake events for probabilistic tsunami hazard assessment. In Global Tsunami Science: Past and Future, Volume I (pp. 3671-3692). Birkhäuser, Cham.

Mai, P. M., \& Beroza, G. C. (2002), A spatial random field model to characterize complexity in earthquake slip. Journal of Geophysical Research: Solid Earth, 107(B11), ESE-10.

Melgar, D., LeVeque, R. J., Dreger, D. S., \& Allen, R. M. (2016), Kinematic rupture scenarios and synthetic displacement data: An example application to the Cascadia subduction zone. Journal of Geophysical Research: Solid Earth, 121(9), 6658-6674.

Melgar, D., \& Hayes, G. P. (2017), Systematic observations of the slip pulse properties of large earthquake ruptures. Geophysical Research Letters, 44(19), 9691-9698.

Melgar, D., \& Hayes, G. P. (2019), The correlation lengths and hypocentral positions of great earthquakes. Bulletin of the Seismological Society of America, 109(6), 2582-2593.

Mena, B., Mai, P. M., Olsen, K. B., Purvance, M. D., \& Brune, J. N. (2010), Hybrid broadband ground-motion simulation using scattering Green's functions: Application to large-magnitude events. Bulletin of the Seismological Society of America, 100(5A), 2143-2162.

Pasyanos, M. E., Masters, T. G., Laske, G., \& Ma, Z. (2014), LITHO1. 0: An updated crust and lithospheric model of the Earth. Journal of Geophysical Research: Solid Earth, 119(3), 2153-2173.

Zhu, L., \& Rivera, L. A. (2002), A note on the dynamic and static displacements from a point source in multilayered media. Geophysical Journal International, 148(3), 619-627. 